\title{
Analytical study of lateral thermal buckling for subsea pipelines with sleeper
}

\author{
Zhenkui Wang ${ }^{\mathrm{a}}$, Yougang Tang, ${ }^{\mathrm{a},{ }^{*}}$, G.H.M. van der Heijden ${ }^{\mathrm{b}}$ \\ ${ }^{\text {a }}$ State Key Laboratory of Hydraulic Engineering Simulation and Safety, Tianjin University, Tianjin 300072, China \\ ${ }^{\mathrm{b}}$ Department of Civil, Environmental and Geomatic Engineering, University College London, London WC1E \\ 6BT, UK
}

\section{Corresponding author: Yougang Tang, tangyougang_td@163.com}

\begin{abstract}
Unburied subsea pipelines operating under high-temperature and high-pressure conditions tend to relieve their axial compressive force by forming lateral buckles. Uncontrolled lateral buckling may lead to pipeline failure. In order to control lateral buckling, a sleeper is often employed as a buckle-initiation technique. In this study, analytical solutions of lateral buckling for unburied subsea pipelines with sleeper are derived. An energy analysis is employed to investigate the stability of the buckled pipeline. The influence of sleeper height and sleeper friction on pipeline buckled configurations and typical lateral buckling behaviour is illustrated and analysed. The results are shown to be in very good agreement with experimental data in the literature. We also discuss the effect of imperfections and conduct an error analysis of one of the main assumptions of the proposed analytical method. Our results show that increasing the height of the sleeper or decreasing the friction between pipeline and sleeper can all be used to decrease the minimum critical temperature difference. However, only the sleeper height is effective in substantially reducing the maximum compressive stress.
\end{abstract}

Keywords: Subsea pipeline; Lateral buckling; Beam-column; Sleeper; Analytical solution.

\section{Introduction}

For the exploitation and transportation of energy resources, subsea pipelines are increasingly being required to operate under high-temperature conditions to ease the flow and prevent solidification of the wax fraction in deep water. The excessive axial compressive force induced by the increase in temperature may lead to lateral buckling for unburied subsea pipelines. Such uncontrolled lateral buckling would cause serious damage to the safety of the pipelines. Consequently, some engineering measures have been employed to prevent subsea pipeline buckling, such as trenching, burying and rock-dumping; or relieving the stress with in-line expansion spools [1]. However, these methods are becoming more and more expensive as the operating temperature increases and as hydrocarbon development moves into deeper water [2].

Thus, an effective and inexpensive method is proposed for the relief of thermal induced axial compressive force, which is to accommodate thermal expansion by artificially inducing the pipeline to buckle in a controlled manner at several controlled locations, rather than to allow it to suffer an uncontrolled, large buckle at one location only. Thermal expansion can be evenly divided into a number of buckles, none of which is subject to too much feed-in from thermal expansion. At these planned locations, a sufficient number of lateral buckles should be triggered at a sufficiently low axial compressive force [3, 4]. Several buckle initiation techniques, which are briefly described by Sinclair et al. [5], have recently been developed to ensure that regular buckles form along the pipeline. Three methods are commonly adopted to promote the reliable formation of lateral buckles and to control the buckle spacing and operating loads, which are snake-lay, sleeper and local weight reduction through distributed buoyancy [6]. A method related to distributed buoyancy is to use discrete buoyancy, such as buoyancy bags, to aid buckle initiation $[3,7,8]$. In this method, a discrete buoyancy, such as an air bag, is only used to initiate lateral buckling, and will be removed once the lateral buckle formation has occurred. Another buckle initiation technique is the zero-radius bend technique proposed by Peek et al. [9]. The advantage in the use of these engineered buckle initiation techniques is that the planned post-buckling configuration is generally more benign than uncontrolled lateral buckles. Consequently, the 
integrity of pipelines within the buckle is improved.

Lateral and upheaval buckling have been studied by previous researchers in the theoretical framework by modelling the pipeline as a beam resting on a rigid seabed [10-17] or on a soft seabed [18-20]. Nonlinear localised lateral buckling of straight pipelines was investigated analytically by Zhu et al. [21] and Wang [22] without the assumption of lateral deformation. On the other hand, small-scale model tests were conducted to understand the mechanism of upheaval buckling of buried pipelines [23, 24] and the properties of man-made initiation techniques to control lateral buckling $[25,26]$. Experimental and numerical investigations were carried out to investigate the buckle interaction between propagation buckling and upheaval or lateral buckling in subsea pipelines by Albermani and Karampour [27, 28]. Moreover, many finite-element analyses have been performed to investigate lateral and upheaval buckling [29-35]. All these methods are employed to investigate lateral buckling or vertical buckling behaviour rather than how to control them.

In recent years, several researches about lateral buckling of subsea pipelines with an initiation technique have been carried out. Simple analytical solutions were given for triggering lateral buckles through applying buoyancy to the pipeline by Peek and Yun [7], which could be applied to a single-point buoyancy load, two-point buoyancy load and distributed buoyancy load over a specified length. Furthermore, the single buoyancy method was further studied by Shi and Wang [3]. The single buoyancy load required to trigger lateral buckles along a pipeline was investigated through analytical methods by Shi and Wang [3]. Analytical solutions were derived based on the first and third lateral buckling mode for a pipeline section with a distributed buoyancy section by Wang et al. [36], Antunes et al. [37] and Li et al. [38].

The use of sleepers as buckle initiation technique does not seem to have attracted much work in the literature. Sinclair et al. [5] conducted a survey of the effect of a sleeper in controlling pipeline lateral buckling and collected operating data on the behaviour of nine pipelines employing sleepers as buckle initiators. Experiments on a scaleddown model were carried out by Silva-Junior et al. [26] and de Oliveira Cardoso et al. [25]. Their studies compare displacements and critical buckling loads of various artificial buckle triggers, including sleepers. Bai et al. [39] studied the applications of dual sleepers as lateral buckling initiators through finite-element modelling. No analytical work appears to exist. Here we propose an analytical model for the study of lateral buckling of subsea pipelines with sleeper.

The survey in [5] shows that the sleeper initiation technique can induce both symmetric (mode 1 or 3 ) and asymmetric (mode 2) buckles (in the classification of Hobbs [10]). The actual mode is driven by the local imperfection introduced during pipe lay. Consequently, it is not possible to predict the buckling mode. However, mode 1 is the most commonly considered lateral buckling mode for pipelines with sleeper according to the surveyed results of Sinclair et al. Thus, the aim of this paper is to derive the analytical solution of the first lateral buckling mode for unburied subsea pipelines with sleeper. Stability of the lateral buckling solutions is analysed by computing the total energy of the pipeline. Parameter studies are carried out to study the effect of sleeper height and sleeper friction on the lateral buckling behaviour. We validate our analytical model by comparing its predictions with the experimental data reported in [25], finding very good agreement. 


\section{Analytical solution}

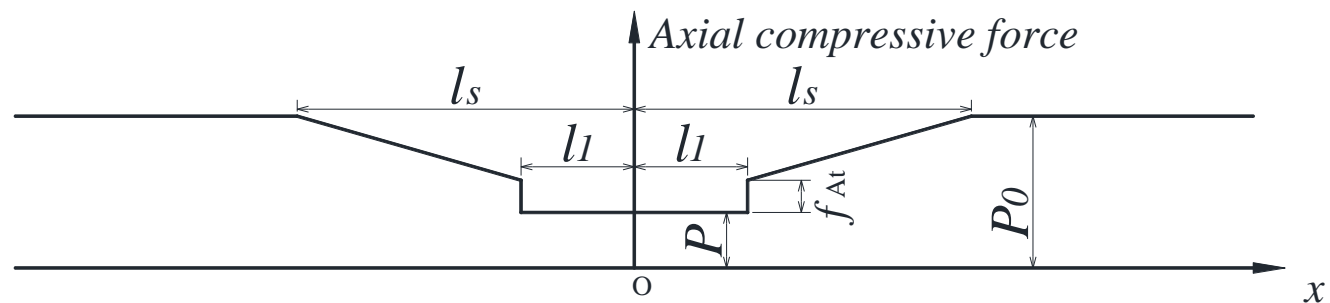

Fig. 1 Axial compressive force distribution.

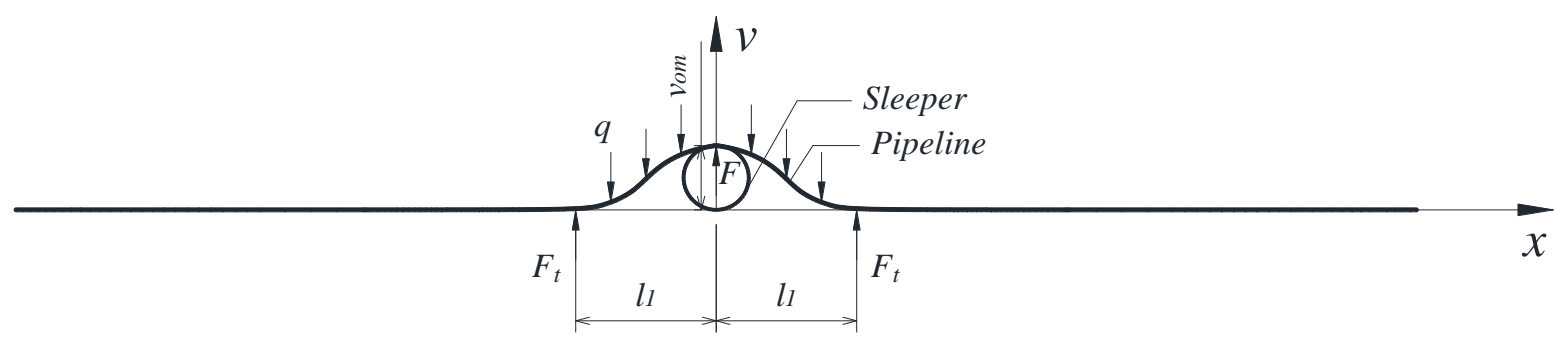

(a)

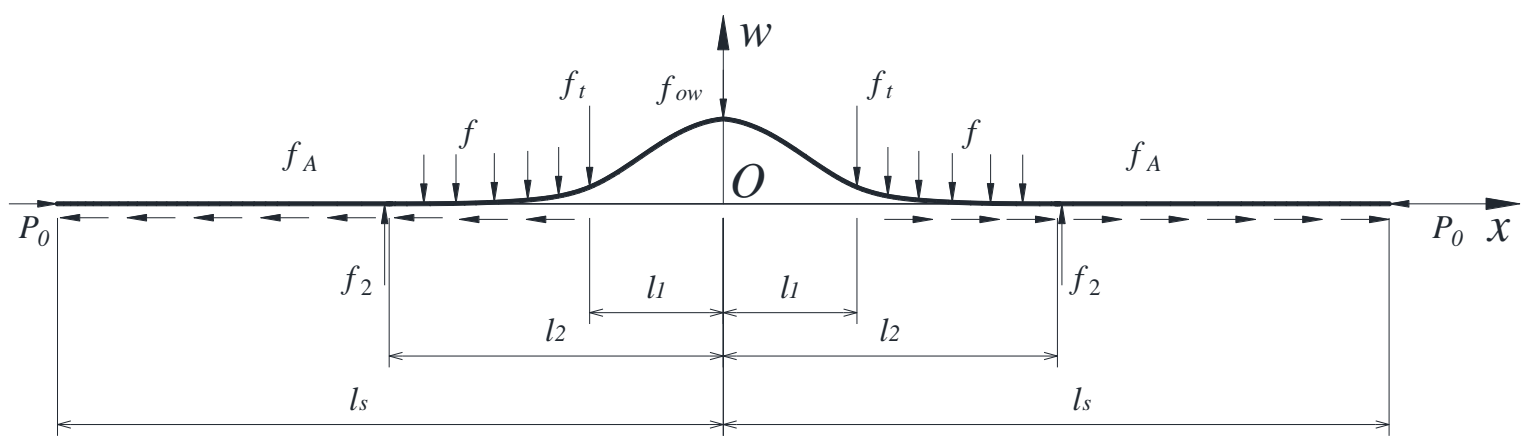

(b)

Fig. 2 Configuration and load distribution. (a) Vertical plane. (b) Lateral plane.

In the process of thermal buckling within a pipeline section that is initially immobilised by axial friction against the seabed a small central segment of pipe will mobilise. As pipe feeds into the buckle the compressive force in the pipe drops, pulling more pipe into the buckle. If the soil resistance for axial movement is constant, say $f_{A}$, then a compressive force will build up in the pipe, increasing linearly with the distance from the touchdown point between pipeline and seabed. At some point this compressive force is sufficient to satisfy the requirement of additional length introduced by the lateral displacement. The end points of this segment are called virtual anchor points. Fig. 1 shows 
the feed-in region of length $2 l_{s}$ within the larger immobilised section of the pipeline together with the typical compressive force variation. $l_{s}$ is sometimes called the slip-length. $P_{0}$ is the axial compressive force at the virtual anchor points.

In practice multiple (independent) localised buckles may form in the immobilised pipe section, especially if it is long. In the following we present a theory for a single localised buckle that applies to each such buckle individually. Fig. 2 illustrates the configuration and load distribution of the first lateral buckling mode for unburied subsea pipelines with sleeper. In the analytical formulations of this mode presented in this section the pipeline is modelled using linear beam-column theory valid for small deflections. The vertical and lateral deflections are therefore essentially independent, coupled only by friction, and we consider each individually.

\subsection{Analytical solution in the vertical plane}

For exposed subsea pipelines the vertical resistance of the seabed is usually greater than the lateral resistance; therefore, the seabed is assumed rigid as a feasible approximation, even for soft soils. Consider a sleeper laid at the middle of the span, as shown in Fig. 2-a. The governing equation for the configuration of the pipeline with sleeper in the vertical plane is

$$
E I \frac{d^{4} v}{d x^{4}}=-q \quad 0 \leq x \leq l_{1}
$$

where $v$ is the vertical deflection, $q$ is the submerged weight per unit length of the pipeline, $E$ is the elastic modulus, $I$ is the moment of inertia, $l_{1}$ is the half span length. Only half of the pipeline needs to be considered owing to the symmetrical configuration and load distribution in the vertical direction.

The general solution of Eq. (1) is

$$
v=-\frac{q}{24 E I} x^{4}+C_{1} x^{3}+C_{2} x^{2}+C_{3} x+C_{4}
$$

By symmetry, the slope of $v$ at $x=0$ must be zero, while the shear force $F$ at $x=0$ comes from the supporting force $2 F$ by the sleeper. In addition, the displacement, slope and moment at $x=l_{1}$ must be zero as well. So the boundary conditions at $x=0$ and $x=l_{1}$ are

$$
\left\{\begin{array}{c}
\frac{d v}{d x}(0)=0 \\
\frac{d^{3} v}{d x^{3}}(0)=\frac{F}{E I} \\
v\left(l_{1}\right)=0 \\
\frac{d v}{d x}\left(l_{1}\right)=0 \\
\frac{d^{2} v}{d x^{2}}\left(l_{1}\right)=0
\end{array}\right.
$$

Combining Eq. (2) and Eq. (3), $C_{1}-C_{4}$ and $F$ can be obtained as

$$
C_{1}=\frac{q l_{1}}{9 E I}, C_{2}=-\frac{q l_{1}^{2}}{12 E I}, C_{3}=0, C_{4}=\frac{q l_{1}^{4}}{72 E I}, F=\frac{2}{3} q l_{1}
$$

Thus, the vertical deflection is

$$
v=\frac{q l_{1}^{4}}{72 E I}\left(-3 \frac{x^{4}}{l_{1}^{4}}+8 \frac{x^{3}}{l_{1}^{3}}-6 \frac{x^{2}}{l_{1}^{2}}+1\right)
$$




$$
v_{o m}=v(0)=\frac{q l_{1}^{4}}{72 E I}
$$

where $v_{o m}$ is the sleeper height.

From Eq. (6) we obtain

$$
l_{1}=\sqrt[4]{\frac{72 E I v_{o m}}{q}}
$$

Thus, the half span length $l_{1}$ can be obtained through Eq. (7) when the sleeper height $v_{\text {om }}$ is given, which will be used in the analysis of the horizontal configuration.

The point contact force between the pipeline and the seabed induced by the uplifted pipeline span at the touchdown point is

$$
F_{t}=q l_{1}-F=1 / 3 q l_{1}
$$

\subsection{Analytical solution in the horizontal plane}

The pipeline consists of three zones in the horizontal plane: one span zone, in which the pipeline is uplifted by the sleeper, and two contacting zones, in which the pipeline contacts the seabed, as shown in Fig. 2-a. We assume that the pipeline always rests on the sleeper, i.e., that the weight of the pipe is always larger than the uplift force. Within the span zone no lateral or axial resistance is provided by the surrounding soil foundation. There is only a concentrated lateral and axial friction force at the touchdown point at $x=l_{1}$, as shown in Fig. 2-b. We assume that the value of the lateral soil resistance is constant for the pipeline lying on the seabed.

With reference to Fig. 1, the axial compressive force distribution $\bar{P}(x)$ can be expressed as

$$
\bar{P}(x)= \begin{cases}P & 0<x<l_{1} \\ P+f_{A t}+f_{A}\left(x-l_{1}\right) & l_{1}<x<l_{s}\end{cases}
$$

Eq. (9)

Note that the axial force is constant $(P)$ in the span region because there is no distributed axial resistance acting on the pipe. For the axial soil resistance (a force per unit length) we can write

$$
f_{A}=\mu_{A} q
$$

where $\mu_{A}$ is the axial friction coefficient between pipeline and seabed. The axial friction force $f_{A t}=\mu_{A} F_{t}$ is induced by the contact vertical force $F_{t}$ at the touchdown point $x=l_{1}$.

The equation governing the horizontal deflection in the span zone is

$$
E I \frac{d^{4} w_{1}}{d x^{4}}+P \frac{d^{2} w_{1}}{d x^{2}}=0 \quad 0 \leq x \leq l_{1}
$$

where, again, by symmetry of the first buckling mode and the load distribution, only half a mode needs to be considered. For the buckled pipeline section lying on the seabed, the governing equation is

$$
E I \frac{d^{4} w_{2}}{d x^{4}}+P \frac{d^{2} w_{2}}{d x^{2}}=-f \quad l_{1} \leq x \leq l_{2}
$$

Eq. (12)

where $f=\mu_{L} q$ is the lateral friction force with $\mu_{L}$ the lateral friction coefficient. Here, for the purpose of determining the deflection $w_{2}$, we have made the simplifying assumption that, despite Eq. (9), the axial compressive force in the entire buckled region $0 \leq x \leq l_{2}$ is constant and equal to the force $P$ at the centre of the buckle. The same approximation was made by Hobbs [10]. 
Let

$$
\lambda^{2}=\frac{P}{E I}
$$

The general solutions of Eq. (11) and Eq. (12) are

$$
\begin{gathered}
w_{1}(x)=A_{1} \cos \lambda x+A_{2} \sin \lambda x+A_{3} x+A_{4}\left(0 \leq x \leq l_{1}\right) \\
w_{2}(x)=B_{1} \cos \lambda x+B_{2} \sin \lambda x+B_{3} x+B_{4}-\frac{f}{2 \lambda^{2} E I} x^{2}\left(l_{1} \leq x \leq l_{2}\right)
\end{gathered}
$$

Eq. (15)

By symmetry, the slope of the deflection $w_{1}$ at $x=0$ must be zero, while the shear force $f_{o w}=\mu_{s} F$ at $x=0$ is induced by the friction force $2 f_{o w}$ between pipeline and sleeper. Here $\mu_{s}$ is the friction coefficient between pipeline and sleeper, also called the sleeper friction. In addition, the displacement, slope and moment at $x=l_{2}$ must be zero. So the boundary conditions at $x=0$ and $x=l_{2}$ are

$$
\left\{\begin{array}{c}
\frac{d w_{1}}{d x}(0)=0 \\
\frac{d^{3} w_{1}}{d x^{3}}(0)+\frac{f_{o w}}{E I}=0 \\
w_{2}\left(l_{2}\right)=0 \\
\frac{d w_{2}}{d x}\left(l_{2}\right)=0 \\
\frac{d^{2} w_{2}}{d x^{2}}\left(l_{2}\right)=0
\end{array}\right.
$$

Eq. (16)

The displacement, slope and bending moment must be continuous at the touchdown point $x=l_{1}$. On the other hand, there is a lateral concentrated friction force $f_{t}=\mu_{L} F_{t}$ at the touchdown point $x=l_{1}$ induced by the point contact force $F_{t}$ at $l_{1}$, so the shear force has a jump at the touchdown point $x=l_{1}$. Thus, the following matching conditions have to be satisfied at $x=l_{1}$ :

$$
\left\{\begin{aligned}
w_{1}\left(l_{1}\right) & =w_{2}\left(l_{1}\right) \\
\frac{d w_{1}}{d x}\left(l_{1}\right) & =\frac{d w_{2}}{d x}\left(l_{1}\right) \\
\frac{d^{2} w_{1}}{d x^{2}}\left(l_{1}\right) & =\frac{d^{2} w_{2}}{d x^{2}}\left(l_{1}\right) \\
\frac{d^{3} w_{1}}{d x^{3}}\left(l_{1}\right) & =\frac{d^{3} w_{2}}{d x^{3}}\left(l_{1}\right)+\frac{f_{t}}{E I}
\end{aligned}\right.
$$

By overall lateral force balance we find

$$
f_{2}=f_{\text {ow }}+f\left(l_{2}-l_{1}\right)+f_{t}
$$

for the point force at $x=l_{2}$, i.e., the end point of the buckled region. This $f_{2}$ is required to prevent a lobe forming in the horizontal plane. (Unlike in the vertical plane, the pipeline is not constrained in the horizontal plane, only resisted by friction, so in general further oscillations or lobes may form. By assuming that these do not form, i.e., that we have a first mode laterally, we effectively consider the unbuckled part of the pipeline for $x>l_{2}$ to provide a rigid support against which the buckled part of the pipeline pushes. This requires the point force $f_{2}$ whose role is completely analogous to that of $F_{t}$ in the vertical plane.)

Axial deformation of the pipeline is governed by the equation

$$
E A \frac{d^{2} u}{d x^{2}}=f_{A} \quad\left(l_{1} \leq x \leq l_{\mathrm{s}}\right)
$$


where $A$ is the cross-sectional area of the pipe. Eq. (18) is solved subject to the slip-length boundary conditions [11]

$$
\left\{\begin{array}{c}
u\left(l_{\mathrm{s}}\right)=0 \\
\frac{d u}{d x}\left(l_{\mathrm{s}}\right)=0
\end{array}\right.
$$

Eq. (19)

giving for the axial displacement

$$
u(x)=\frac{f_{A}}{2 E A}\left(x-l_{\mathrm{s}}\right)^{2}
$$

Eq. (20)

This result will be used later when computing the total potential energy of a buckled pipe solution. Finally, by axial force balance, we have

$$
P_{0}=P+f_{A}\left(l_{s}-l_{1}\right)+f_{A t}
$$

Eq. (21)

We now use compatibility between axial and lateral deformation in the feed-in zone $0 \leq x \leq l_{s}$ to derive a relationship between the axial compressive force $P$ in the uplifted section of the pipe and the temperature difference $T_{0}$, between the fluid flowing inside the pipe and the environment, that causes the buckling. Compatibility can be expressed as

$$
u_{1}=u_{2}
$$

Eq. (22)

where $u_{1}$ is the length of axial expansion within the pipeline section $0<x<l_{s}$ due to high pressure and high temperature. $u_{2}$ is the geometric shortening, which allows for the additional length introduced by the lateral displacement. Eq. (22) simply states that, since there are virtual anchor points at distance $l_{\mathrm{s}}$ from the centre of the pipe, the extra length of pipe in the buckle must come from axial expansion of the mobilised segment of pipe.

We have

$$
u_{1}=\int_{0}^{l_{s}} \frac{\Delta \bar{P}(x)}{E A} d x
$$

where $\Delta \bar{P}(x)$ is the amount of decrease of axial compressive force along the pipeline after the pipeline buckles, which, from Eq. (9), is given by

$$
\Delta \bar{P}(x)= \begin{cases}P_{0}-P & 0<x<l_{1} \\ f_{A}\left(l_{s}-x\right) & l_{1}<x<l_{S}\end{cases}
$$

Thus, we find

$$
u_{1}=\frac{f_{A}\left(l_{s}-l_{1}\right)^{2}}{2 E A}+\frac{\left(P_{0}-P\right) l_{1}}{E A}
$$

Meanwhile, for $u_{2}$ we have

$$
u_{2}=\frac{1}{2} \int_{0}^{l_{1}}\left(\frac{d w_{1}}{d x}\right)^{2} d x+\frac{1}{2} \int_{l_{1}}^{l_{2}}\left(\frac{d w_{2}}{d x}\right)^{2} d x
$$

Combining Eq. (22) and Eq. (25), we obtain the following equation

$$
l_{s}=\sqrt{\frac{1}{3} l_{1}^{2}+\frac{2 E A u_{2}}{f_{A}}}
$$


from which, with the use of Eq. (21) and Eq. (8), we finally obtain

$$
P_{0}=P+f_{A}\left(\sqrt{\frac{1}{3} l_{1}^{2}+\frac{2 E A u_{2}}{f_{A}}}-\frac{2}{3} l_{1}\right)
$$

Within the range of linear elastic response this compressive force $P_{0}$ can be written as

$$
P_{0}=E A \alpha T_{0}
$$

where $\alpha$ is the coefficient of linear thermal expansion. $T_{0}$ is here the total temperature difference, which is composed of the initial temperature difference and the equivalent temperature difference generated by internal pressure.

So given $T_{0}$, Eq. (29), Eq. (28) and Eq. (26) (with Eq. (14) and Eq. (15) inserted) can be solved in conjunction with Eq. (16) and Eq. (17) to obtain $P, l_{2}$ and the coefficients $A_{1}-A_{4}$ and $B_{1}-B_{4}$, and hence the lateral deflection $w_{1}, w_{2}$.

The bending moment $M_{m}$ along the buckled pipeline can be obtained by

$$
M_{m}=E I \frac{d^{2} w}{d x^{2}}
$$

where $w$ stands for either $w_{1}$ or $w_{2}$. Thus, the corresponding bending stress $\sigma_{M}$ along the buckled pipeline is

$$
\sigma_{M}=\frac{M_{m} D}{2 I}
$$

where $D$ is the external diameter of the pipe. The maximum stress $\sigma_{m}$ along the pipeline induced by axial compressive force $P$ and bending moment $M_{m}$ can be obtained from the following expression

$$
\sigma_{m}=\frac{P}{A}+\frac{M_{m} D}{2 I}
$$

Eq. (32)

\section{Analytical results}

\subsection{Relationship between $\lambda$ and $l_{2}$}

The solution for the pipeline deflection $w_{1}, w_{2}$ can be presented in semi-explicit form by using Eq. (16) and Eq. (17) to express the coefficients $A_{\mathrm{i}}, B_{\mathrm{i}}$ in terms of $\lambda, l_{2}$ and all the parameters of the problem (see the Appendix). For the relationship between $\lambda$ and $l_{2}$ we then still need to solve a transcendental equation. For pipelines without sleeper $\left(v_{o m}=0\right)$ the value of $\lambda l_{2}$ is 4.4934. However, for pipelines with sleeper $\left(v_{o m}>0\right)$ the value of $\lambda l_{2}$ changes with sleeper height $v_{o m}$ and friction coefficient $\mu_{s}$ between pipeline and sleeper. For $v_{o m}=0.5 \mathrm{~m}$ and $\mu_{s}=0.1$, the relationship between $l_{2}$ and $\lambda$ is given graphically in Fig. 3-a. By using Eq. (28) and Eq. (29) we can also obtain the relationship between $l_{2}$ and the temperature difference $T_{0}$. The result is shown in Fig. 3-b. Note that conversion from $P$ to $T_{0}$ leads to a fold in the solution curve: a single temperature corresponds to two different central compressive forces. This non-uniqueness of solutions highlights the need for a stability analysis, which we perform by means of an energy analysis in which we determine the total energy involved in thermal pipeline buckling. 


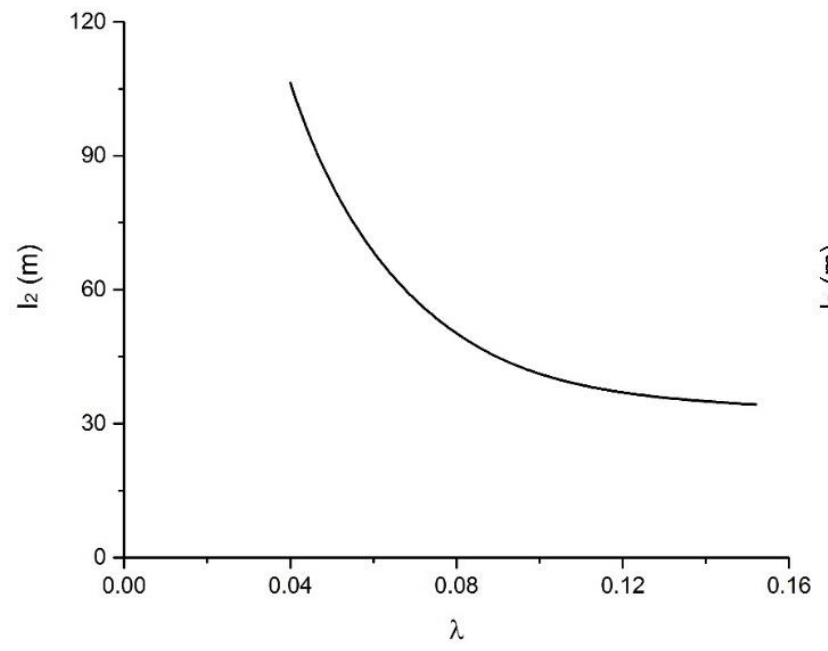

(a)

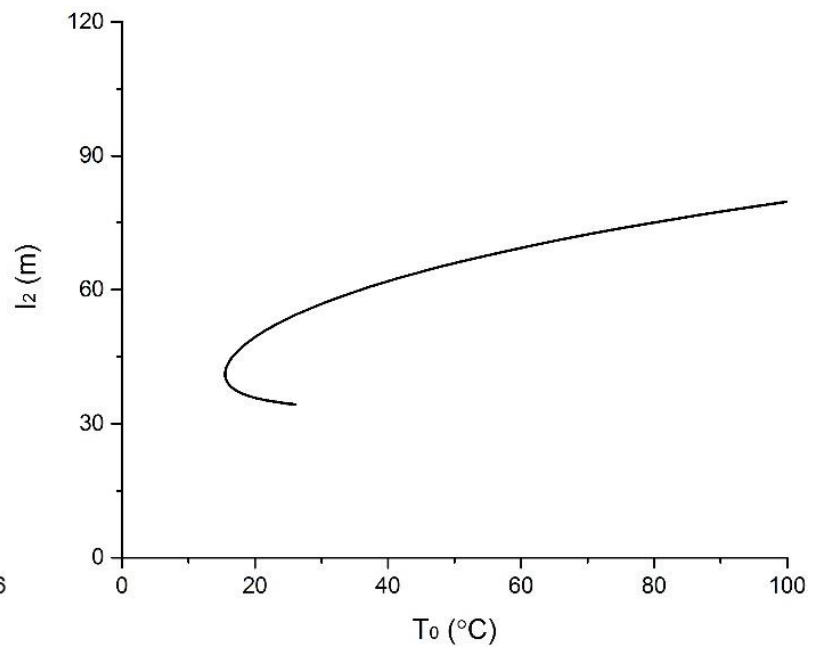

(b)

Fig. 3 Length of the buckled region $l_{2}$. (a) Relationship between $l_{2}$ and $\lambda$. (b) Relationship between $l_{2}$ and $T_{0}$.

$$
v_{\text {om }}=0.5 \mathrm{~m} . \mu_{\mathrm{s}}=0.1 \text {. }
$$

\subsection{Energy analysis of a typical buckling path}

The typical relationship between lateral buckling amplitude $w_{m}$ and total temperature difference $T_{0}$ for a typical solution with sleeper is shown in Fig. 4. The significant point $m$ along the post-buckling path corresponds to the minimum critical temperature difference $T_{m} . T_{m}=15.5121{ }^{\circ} \mathrm{C}$ for this case. For $T_{0}>T_{m}$ two solution branches exist, which will be referred to as $m-b$ and $m-c$, as shown in Fig. 4. We shall compare the energies.

The total potential energy relating to the buckled pipeline (in the feed-in region $0 \leq x \leq l_{s}$ ) is given by

$$
V=V_{1}+V_{2}+V_{3}+V_{4}
$$

The bending strain energy $V_{1}$ can be expressed as

$$
V_{1}=\frac{1}{2} E I \int_{0}^{l_{1}}\left(\frac{d^{2} w_{1}}{d x^{2}}\right)^{2} d x+\frac{1}{2} E I \int_{l_{1}}^{l_{2}}\left(\frac{d^{2} w_{2}}{d x^{2}}\right)^{2} d x
$$

The energy loss $V_{2}$ due to lateral soil resistance is

$$
V_{2}=\int_{l_{1}}^{l_{2}}\left|f w_{2}(x)\right| d x+\left|f_{o w} w_{1}(0)\right|+\left|f_{t} w_{1}\left(l_{1}\right)\right|
$$

The energy loss $V_{3}$ due to axial soil resistance is

$$
V_{3}=\int_{l_{1}}^{l_{s}}\left|f_{A} u(x)\right| d x+\left|f_{A t} u\left(l_{1}\right)\right|
$$

The axial compressive strain energy $V_{4}$ is

$$
V_{4}=\frac{1}{2 E A} \int_{0}^{l_{s}} \bar{P}(x)^{2} d x
$$

while the total potential energy of the straight pipeline, namely before buckling, is given by

$$
V_{i}=\frac{1}{2 E A} \int_{0}^{l_{s}} P_{0}^{2} d x
$$




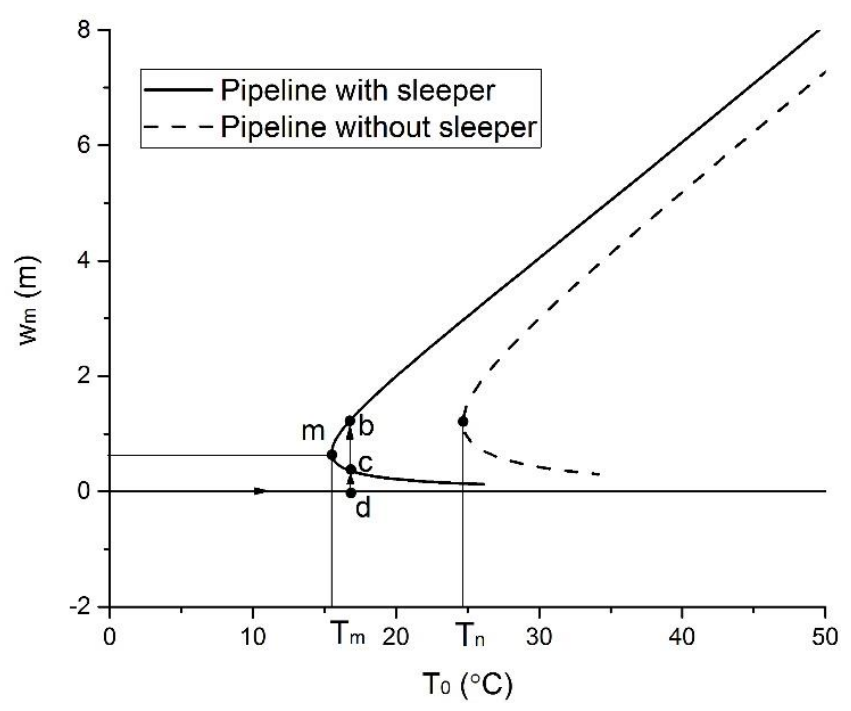

Fig. 4 Typical buckling path. $v_{o m}=0.5 \mathrm{~m} . \mu_{s}=0.1$.

When $T_{0}$ is lower than $T_{m}$ only the trivial state $\left(w_{m}=0\right)$ exists and no lateral buckling occurs. The pipeline remains in the vertical plane. However, when $T_{0}$ is larger than $T_{m}$, two lateral buckling states exist. Take $T_{0}=$ $16.75{ }^{\circ} \mathrm{C}$, for example. When $T_{0}$ reaches $16.75^{\circ} \mathrm{C}$, the pipeline will remain unbuckled in the absence of a disturbance or imperfection, corresponding to point $d$ in Fig. 4. However, the deformed states $b$ and $c$ are available as well and a sufficiently large disturbance may cause a jump from $d$ to one of these buckled states. From the fact that the post-buckling branch does not intersect the trivial branch we deduce that there is no critical temperature $T_{0}$ at which the pipe starts gradually to move sideways on the sleeper. Instead, the pipe will lift off the sleeper and then, at slightly higher $T_{0}$, fall sideways back onto the sleeper. Thus a jump occurs from $d$ to a point near $b$ or $c$ in Fig. 4 . The total energy is calculated through Eq. (33) to determine the relative stability of the two branches. Since $l_{s}$ depends on the precise shape of the solution, energies $V$ for different solutions are not directly comparable. For a meaningful comparison we ensured pipes had equal length by adding extra length of (axially strained) pipe as necessary.

The total energy of branches $m-b$ and $m-c$ for the lateral post-buckling state are denoted by $V_{b}$ and $V_{c}$, respectively. $V_{i b}$ and $V_{i c}$ are the total potential energies of the unbuckled pipeline with sleeper of corresponding length $l_{s} . V_{b} / V_{i b}$ and $V_{c} / V_{i c}$ are illustrated in Fig. 5. We see that all the values of $V_{b} / V_{i b}$ are less than those of $V_{c} / V_{i c}$, which means that the branch $m-b$ is more stable than branch $m$-c. In addition, the value of $V_{c} / V_{i c}$ first increases slightly and then decreases with increasing temperature difference, while all the values of $V_{c} / V_{i c}$ are larger than 1 , which means that branch $m$-c is less stable than the trivial solution. The value of $V_{b} / V_{i b}$ decreases with increasing temperature difference, which means that the branch $m-b$ becomes more stable with increasing temperature difference. $V_{b} / V_{i b}=1$ when the temperature difference reaches $T_{e}=16.58^{\circ} \mathrm{C}$. For $T_{0}<T_{e}, V_{b} / V_{i b}$ is bigger than 1, which means that the trivial solution is more stable. For $T_{0}>T_{e}, V_{b} / V_{i b}$ is smaller than 1 , which means that the branch $m-b$ is more stable than the trivial state.

Fig. 4 also displays the buckling curve for the same pipeline without sleeper. We see that for $T_{0}>T_{n}$ two deformed states are available and an energy analysis shows that again the upper branch contains solutions with lower energy than the trivial state. A pipeline without sleeper, therefore, would likely jump into a buckled state under a sufficiently large disturbance when $T_{0}$ becomes larger than $T_{n}$. This buckling would be sudden, without any warning signs, and could happen at any point of a long immobilised pipeline. This potentially dangerous scenario is avoided by using a sleeper, which forces the pipeline into a deformed state at a specific point and at lower $T_{0}$ before the critical temperature $T_{n}$ is reached. We see in Fig. 4 that the amplitude of lateral deflection will be larger in the case of a sleeper (at the same temperature difference $T_{0}$ ). The effect of the sleeper will be investigated in more detail 
in Section 3.3.

In Fig. 6 we analyse the composition of the total potential energy of the buckled pipeline, namely branch $m-b$. Both $V_{1} / V$ and $V_{2} / V$ first increase slightly and then reduce to less than $10 \%$ with increasing temperature difference. However, $V_{3} / V$ increases rapidly with temperature. We conclude that for large temperature difference the main energy loss is due to axial soil resistance. $V_{4} / V$ decreases rapidly to $50 \%$ at about $25{ }^{\circ} \mathrm{C}$ first and then decreases slightly with increasing temperature difference. Thus, the total potential energy relating to the buckled pipeline consists mainly of $V_{3}$ and $V_{4}$.

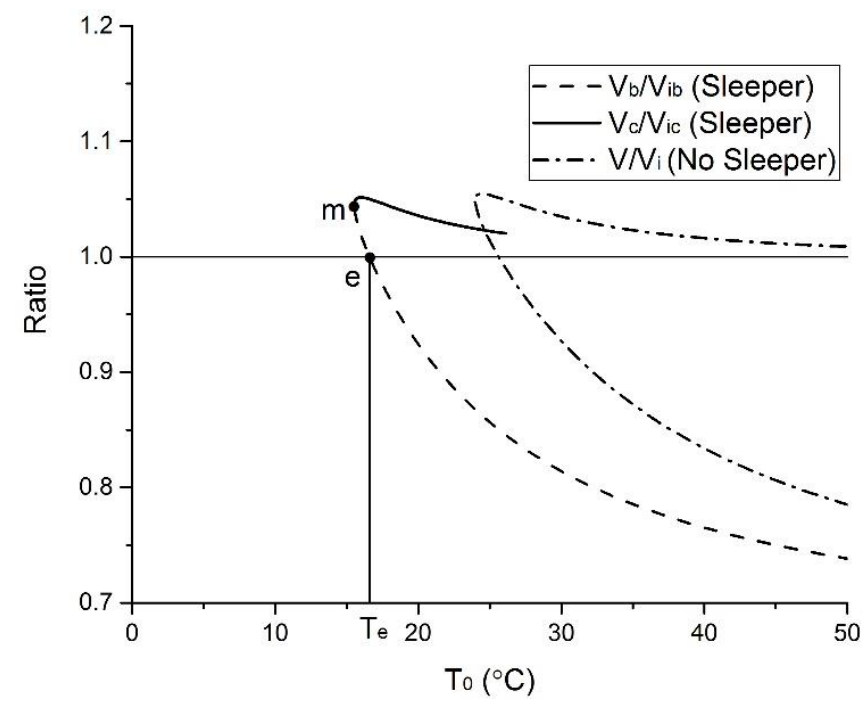

Fig. 5 Ratio of the energy between the buckled state and the pre-buckling state. $v_{o m}=0.5 \mathrm{~m} . \mu_{s}=0.1$.

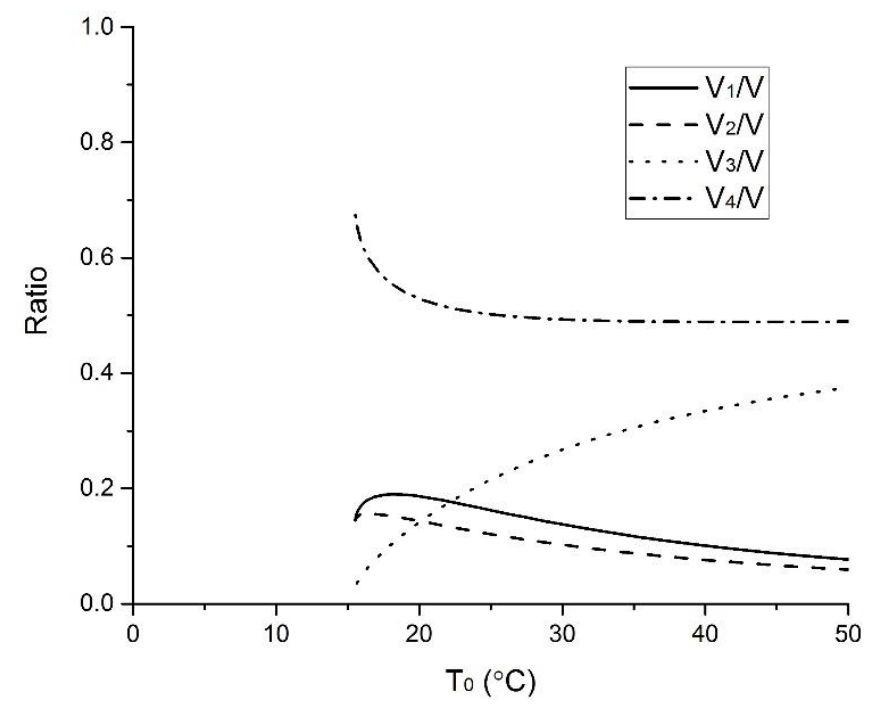

Fig. 6 Composition of the total potential energy of the buckled pipeline. $v_{o m}=0.5 \mathrm{~m} . \mu_{s}=0.1$.

\subsection{Influence of the sleeper on lateral buckling}

Table 1. Design parameters

\begin{tabular}{ccc}
\hline Parameters & Values & Unit \\
\hline External diameter $D$ & 323.9 & $\mathrm{~mm}$ \\
Wall thickness $t$ & 12.7 & $\mathrm{~mm}$ \\
Elastic modulus $E$ & 206 & $\mathrm{GPa}$
\end{tabular}




\begin{tabular}{ccc} 
Steel density $\rho$ & 7850 & $\mathrm{~kg} / \mathrm{m}^{3}$ \\
Coefficient of thermal expansion $\alpha$ & $1.1 \times 10^{-5}$ & $/{ }^{\circ} \mathrm{C}$ \\
Lateral friction coefficient $\mu_{L}$ & 0.5 & --- \\
Axial friction coefficient $\mu_{A}$ & 0.5 & --- \\
Sleeper friction $\mu_{S}$ & $0.1 / 0.2 / 0.3$ & --- \\
Sleeper height $v_{\text {om }}$ & $0.1 / 0.3 / 0.5$ & $\mathrm{~m}$ \\
\hline
\end{tabular}

In this section, a typical pipeline with sleeper resting on the seabed is analysed for the first lateral buckling mode. The deformed shapes and bending stresses along the pipeline with different sleeper height $v_{o m}$ and sleeper friction $\mu_{s}$ under the same temperature are analysed and discussed. The sleeper changes the properties of pipeline lateral buckling, such as the lateral buckling amplitude $w_{m}$, the half-length of buckled section $l_{2}$, the axial compressive force $P$, the axial expansion displacement $u_{1}$, the length of feed-in zone $l_{s}$ and the maximum axial compressive stress $\sigma_{m}$. We demonstrate this sleeper effect by employing the analytical formulation developed in Section 2 taking the parameters in Table 1 as a realistic case study. In this section, all the analysis is based on branch $\mathrm{m}$-b, namely the stable branch.

\subsubsection{Influence of the sleeper on the configuration}

The deformed shapes and the corresponding bending stresses $\sigma_{M}$ along the buckled pipeline with different sleeper height under the same operating temperature difference are presented in Fig. 7-a and Fig. 7-b, respectively. In Fig. 7a it is seen that both the buckled region and the lateral deflection for the buckled pipeline with sleeper are larger than those without sleeper, and grow with increasing sleeper height. We also note that the lateral displacement amplitude increases with increasing sleeper height. As for the bending stress $\sigma_{M}$, there are two locations of maximum bending stress along the buckled pipeline for a given sleeper height. For the chosen temperature difference $T_{0}=25^{\circ} \mathrm{C}$, the maximum (compressive) bending stress at $x=0 \mathrm{~m}$ for the pipeline with sleeper height $v_{o m}=0.5 \mathrm{~m}$ is a little smaller than that without sleeper. However, for the pipeline with sleeper height $v_{o m}=0.1 \mathrm{~m}$ and $v_{o m}=0.3 \mathrm{~m}$, the maximum (compressive) bending stress at $x=0 \mathrm{~m}$ is larger than that without sleeper. Generally, for larger $T_{0}$, the maximum (compressive) bending stress for a pipeline with sleeper is smaller than that for a pipeline without sleeper (see Fig. 14). For pipelines with different sleeper height, the maximum (compressive) bending stress decreases with increasing sleeper height. The sleeper height does not have much effect on the maximum (tensile) bending stress near $x=40 \mathrm{~m}$, which is a little smaller than that without sleeper, while the maximum value of bending stress $\sigma_{M}$ at $x=$ $0 \mathrm{~m}$ is larger (in absolute value) than that at $x=40 \mathrm{~m}$.

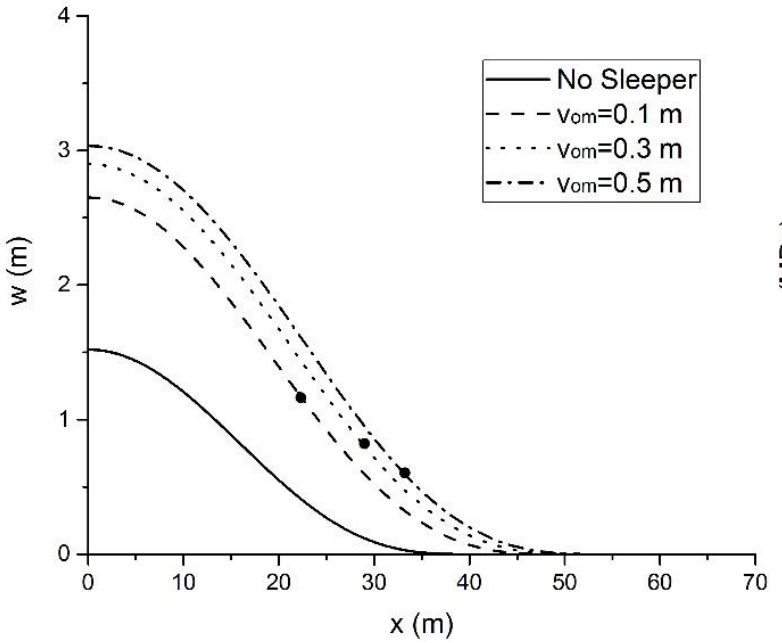

(a)

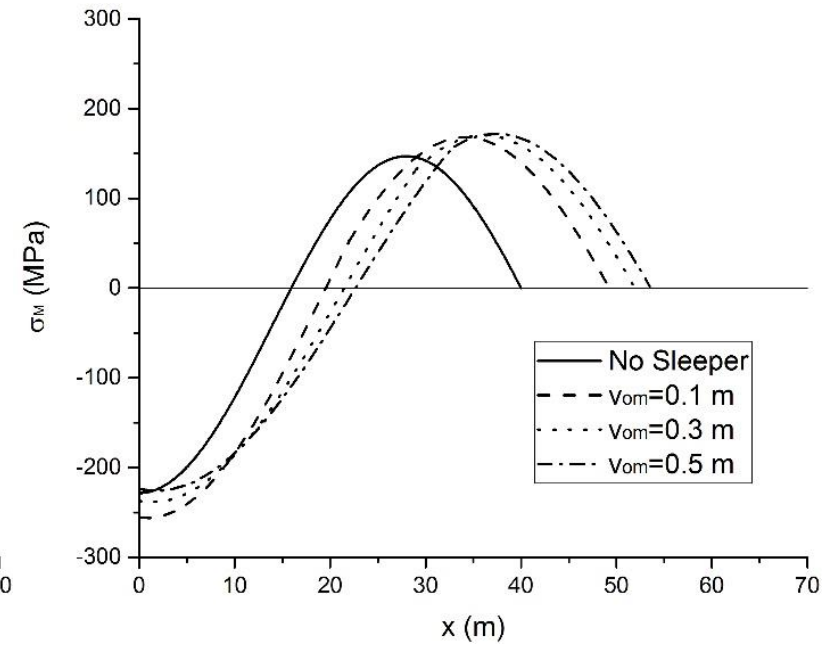

(b)

Fig. 7 The influence of sleeper height. (a) Deformed shapes. (b) Bending stress. $\mu_{s}=0.1 . T_{0}=25^{\circ} \mathrm{C}$. The dot represents the touchdown point $x=l_{1}$. 


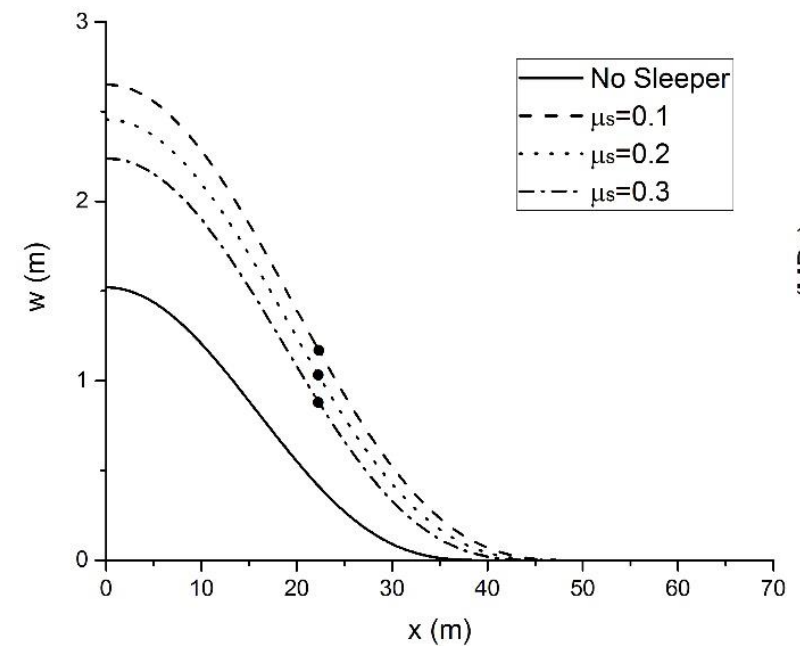

(a)

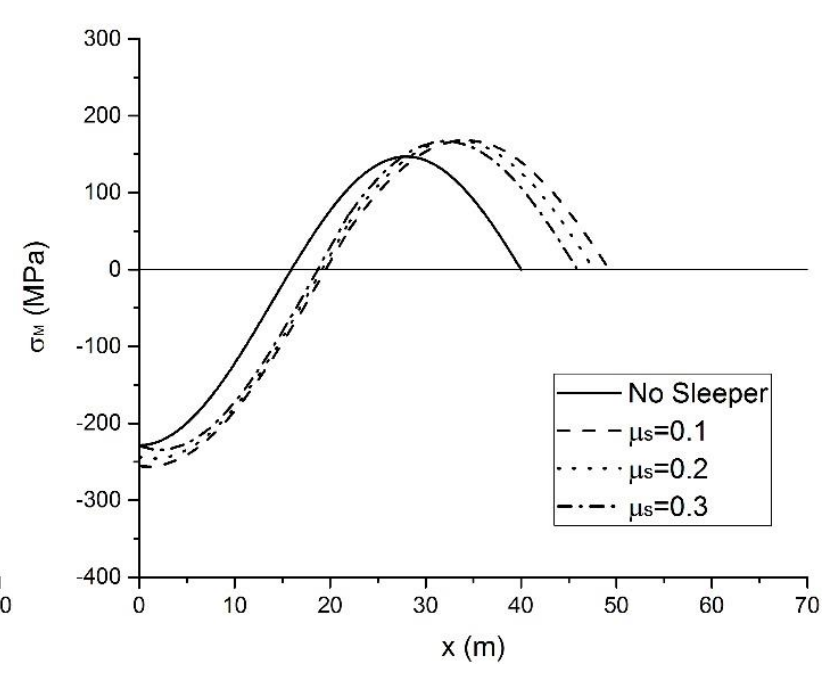

(b)

Fig. 8 The influence of sleeper friction. (a) Deformed shapes. (b) Bending stress. $v_{o m}=0.1 \mathrm{~m} . T_{0}=25^{\circ} \mathrm{C}$. The dot represents the touchdown point $x=l_{1}$.

The deformed shapes and the corresponding bending stress $\sigma_{M}$ along the buckled pipeline with different values of sleeper friction under the same operating temperature difference are presented in Fig. 8-a and Fig. 8-b, respectively. In Fig. 8-a the lateral deflection of the pipeline with sleeper increases with decreasing sleeper friction and is larger than that without sleeper. The sleeper friction has a bigger effect on the region of lateral deflection that is closer to the sleeper. However, the sleeper friction does not have much effect on the size of the buckled region $l_{2}$. From Fig. 8-b we see that the maximum value of the bending stress $\sigma_{M}$ for a pipeline with sleeper friction $\mu_{s}=0.1$ and $\mu_{s}=$ 0.2 is larger than that without sleeper for the chosen temperature difference $T_{0}=25^{\circ} \mathrm{C}$. Generally, for larger $T_{0}$, the maximum (compressive) bending stress for a pipeline with sleeper is smaller than that for a pipeline without sleeper (see Fig. 14). For pipelines with different sleeper friction, the maximum (compressive) bending stress decreases with increasing sleeper friction. The sleeper friction does not have much effect on the bending stress $\sigma_{M}$. The maximum value of the bending stress $\sigma_{M}$ at $x=0 \mathrm{~m}$ is larger (in absolute value) than that at $x=40 \mathrm{~m}$.

\subsubsection{Influence of the sleeper on the post-buckling behaviour}

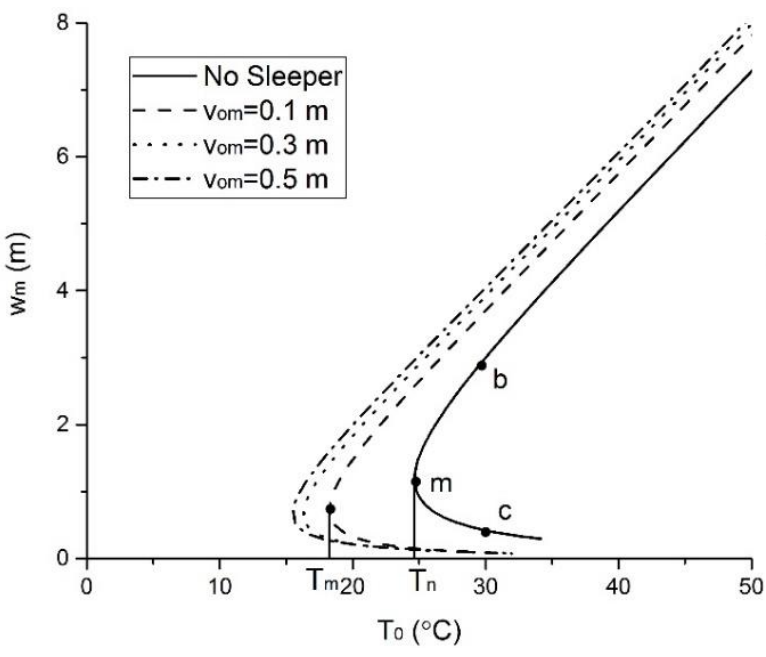

(a)

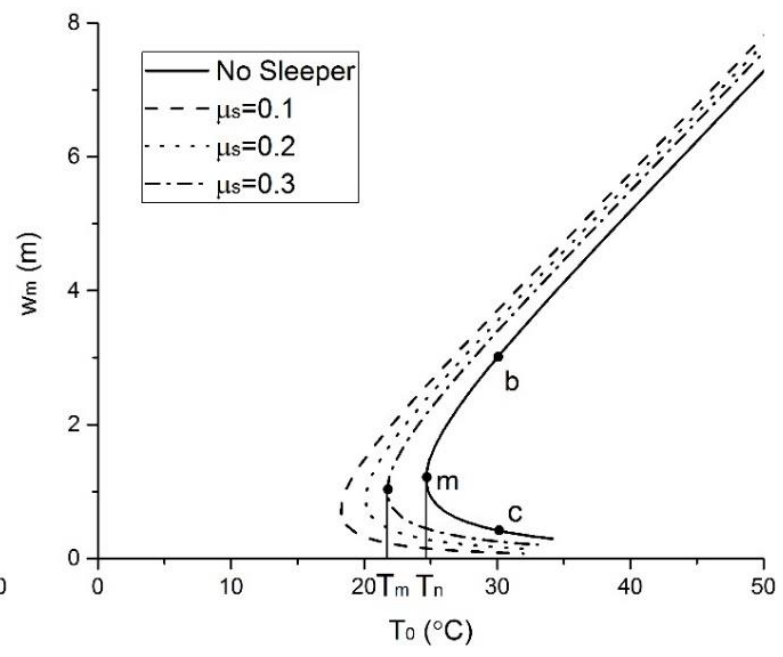

(b)

Fig. 9 Lateral displacement amplitude $w_{m}$. (a) Different sleeper height $v_{o m} . \mu_{s}=0.1$. (b) Different sleeper friction $\mu_{s} . v_{o m}=0.1 \mathrm{~m}$.

The typical relationships between lateral displacement amplitude $w_{m}$ and total temperature difference $T_{0}$ with 
different values of sleeper height $v_{o m}$ and sleeper friction $\mu_{s}$ are shown in Fig. 9-a and Fig. 9-b, respectively. We present results for $0<T_{0}<50^{\circ} \mathrm{C}$ but note that deflections for large $T_{0}$ are not reliable and need a large-deflection theory (for instance, for $T_{0}=40^{\circ} \mathrm{C}$ the deflection $w_{m}$ is about $6 \mathrm{~m}$, which corresponds to 18 pipe diameters). It is seen from Fig. 9-a that $T_{m}$ decreases with increasing sleeper height $v_{o m}$ for a given sleeper friction $\mu_{s}$. Also, $T_{m}$ for pipelines with sleeper are all smaller than those for pipelines without sleeper. However, $T_{m}$ increases with increasing sleeper friction $\mu_{s}$ for a given sleeper height $v_{o m}$, as seen in Fig. 9-b. The minimum critical temperature difference $T_{m}$ for a sleeper with $\mu_{s}=0.3$ is close to that for a pipeline without sleeper. $T_{m}$ will be larger than that for a pipeline without sleeper with increasing sleeper friction $\mu_{s}$. Thus, it is an effective method to reduce the value of sleeper friction $\mu_{s}$ in order to trigger lateral buckling. For given sleeper height $v_{o m}$ and sleeper friction $\mu_{s}$ the lateral displacement amplitude $w_{m}$ increases with increasing total temperature difference $T_{0}$. The lateral displacement amplitude $w_{m}$ increases with increasing sleeper height $v_{o m}$ and decreases with increasing sleeper friction $\mu_{s}$ under the same temperature difference $T_{0}$, and is bigger than that of a pipeline without sleeper.

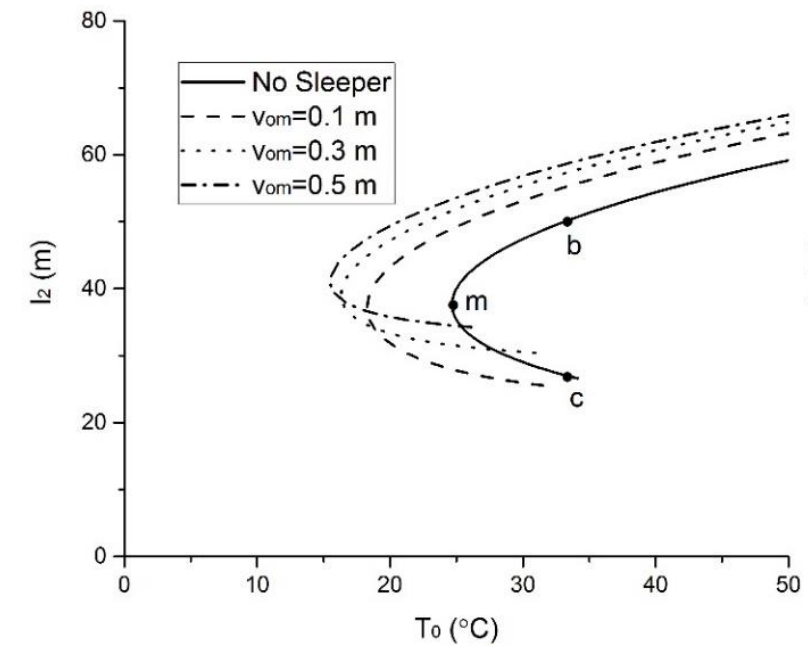

(a)

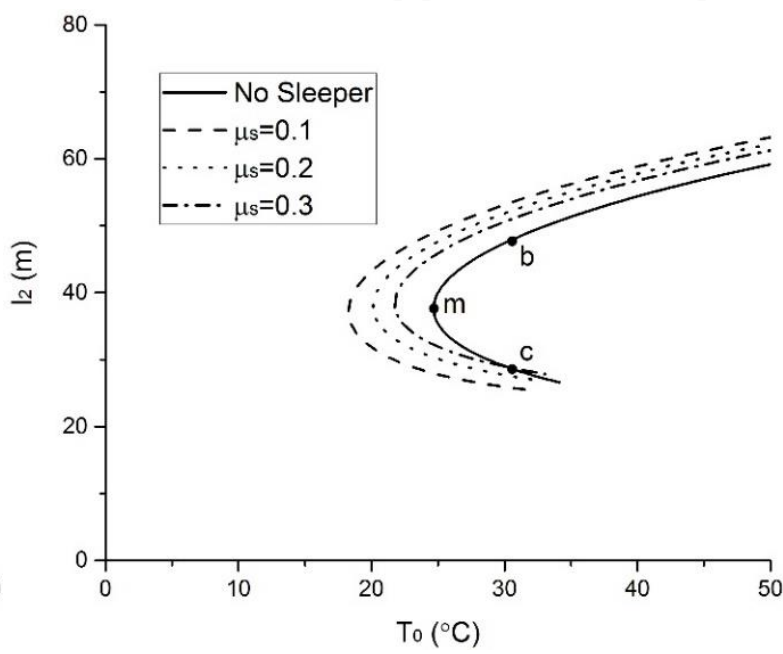

(b)

Fig. 10 Half-length $l_{2}$ of the buckled section. (a) Different sleeper height $v_{o m} \cdot \mu_{s}=0.1$. (b) Different sleeper friction $\mu_{s} . v_{o m}=0.1 \mathrm{~m}$.

The typical relationships between the half-length of the buckled section $l_{2}$ and the total temperature difference $T_{0}$ with different values of sleeper height $v_{o m}$ and sleeper friction $\mu_{s}$ are shown in Fig. 10-a and Fig. 10-b, respectively. For given sleeper height $v_{o m}$ and sleeper friction $\mu_{s}$ the half-length $l_{2}$ increases with increasing total temperature difference $T_{0}$, as seen in Fig. 10. But the rate of increase reduces with increasing $T_{0}$ and the half-length $l_{2}$ increases with increasing sleeper height $v_{o m}$ and decreases with increasing sleeper friction $\mu_{s}$ under the same temperature difference $T_{0}$, and is bigger than that of a pipeline without sleeper. 


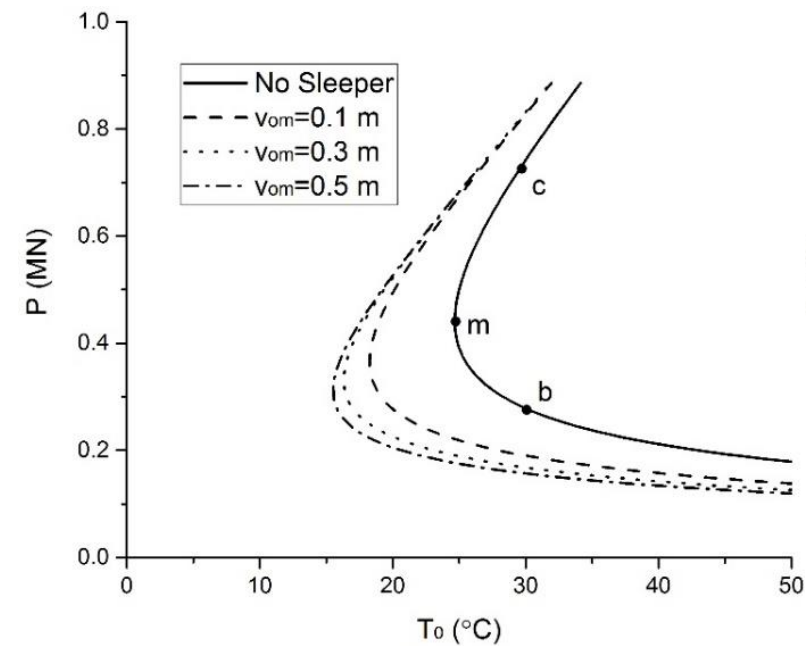

(a)

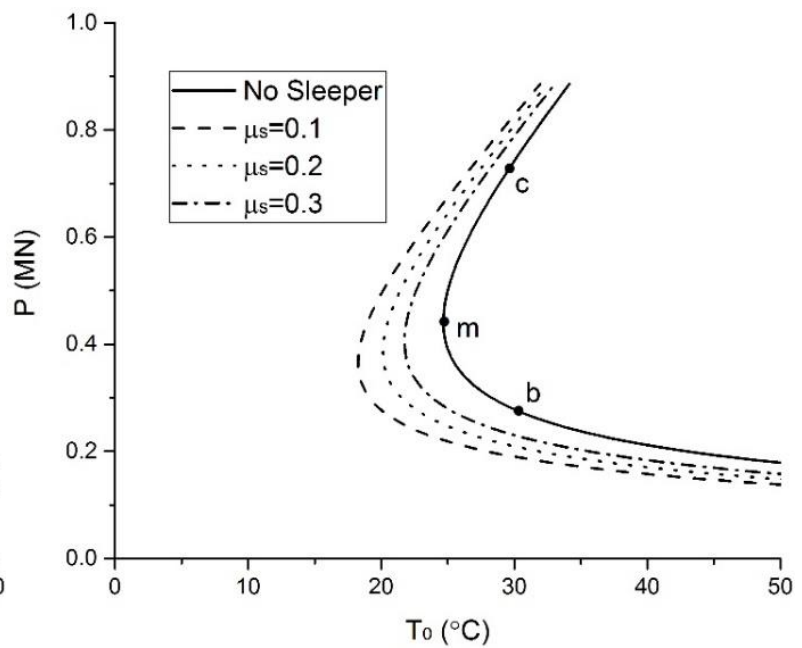

(b)

Fig. 11 Axial compressive force P. (a) Different sleeper height $v_{o m} . \mu_{s}=0.1$. (b) Different sleeper friction $\mu_{s}$.

$$
v_{\text {om }}=0.1 \mathrm{~m} \text {. }
$$

The relationships between axial compressive force $P$ and total temperature difference $T_{0}$ with different values of sleeper height $v_{o m}$ and sleeper friction $\mu_{s}$ are shown in Fig. 11-a and Fig. 11-b, respectively. It is seen from Fig. 11 that the axial compressive force $P$ decreases with increasing total temperature difference $T_{0}$ for given sleeper height $v_{o m}$ and sleeper friction $\mu_{s}$. This shows that the process of lateral buckling results in an axial compressive force reduction under increasing $T_{0}$. The axial compressive force $P$ decreases with increasing sleeper height $v_{o m}$ and increases with increasing sleeper friction $\mu_{s}$ under the same temperature difference $T_{0}$. Thus, the pipeline will be more stable because of smaller axial compressive force $P$ in the post-buckling stage for larger sleeper height $v_{o m}$ and smaller sleeper friction $\mu_{s}$. However, the free span length $\left(2 l_{1}\right)$ will increase with increasing sleeper height $v_{o m}$ and since a pipeline with larger span length has lower natural frequency, which will be closer to the fluid vortex frequency, this will more easily lead to vortex-induced vibration [40]. So both lateral buckling and vortex-induced vibration should be considered for the selection of an appropriate sleeper height $v_{o m}$.

The relationships between axial expansion displacement $u_{1}$ and total temperature difference $T_{0}$ with different values of sleeper height $v_{o m}$ and sleeper friction $\mu_{s}$ are shown in Fig. 12-a and Fig. 12-b, respectively. It is seen from Fig. 12 that the axial expansion displacement $u_{1}$ increases with increasing total temperature difference $T_{0}$ for given sleeper height $v_{o m}$ and sleeper friction $\mu_{s}$. The axial expansion displacement $u_{1}$ is larger for larger sleeper height $v_{o m}$ or smaller sleeper friction $\mu_{s}$. Because the lateral displacement will increase with increasing sleeper height $v_{o m}$ (see Fig. 7-a) and decrease with increasing sleeper friction $\mu_{s}$ (see Fig. 8-a), which requires more axial feed-in displacement for larger sleeper height $v_{o m}$ and smaller sleeper friction $\mu_{s}$, and the axial feed-in displacement comes from the axial expansion of the pipeline, the length of feed-in zone $l_{s}$ also increases with increasing sleeper height $v_{o m}$ and decreases with increasing sleeper friction $\mu_{s}$ under the same temperature difference, as shown in Fig. 13. We also see from Fig. 13 that the length of the feed-in zone $l_{s}$ increases with increasing total temperature difference $T_{0}$ for given sleeper height $v_{o m}$ and sleeper friction $\mu_{s}$. Thus, a series of sleepers can be artificially installed at planned locations to control the length of the feed-in zone $l_{s}$, which can be used to mitigate lateral buckle formation by adjusting the intervals between sleepers. 


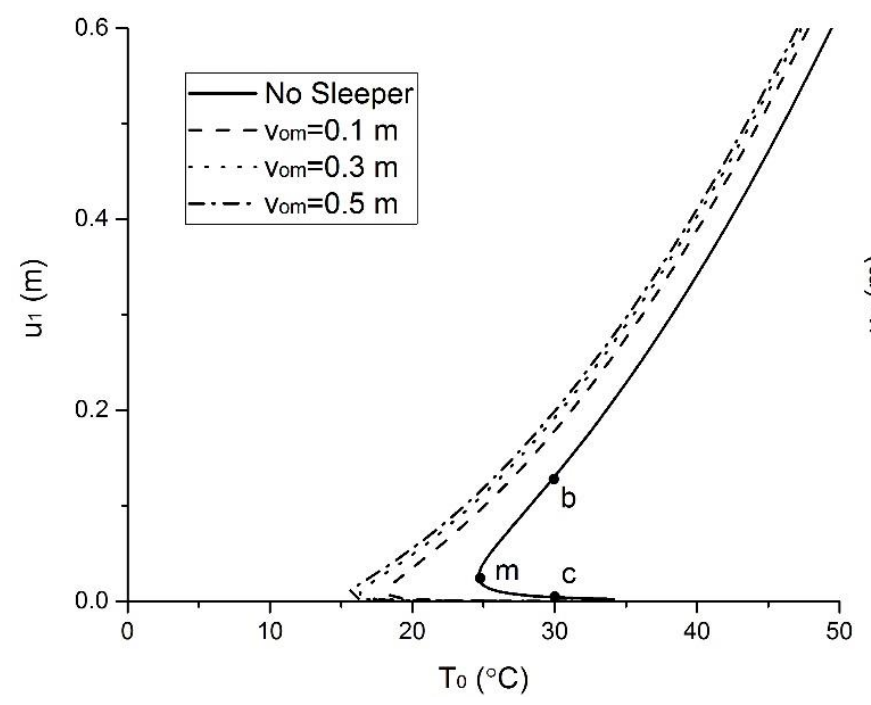

(a)

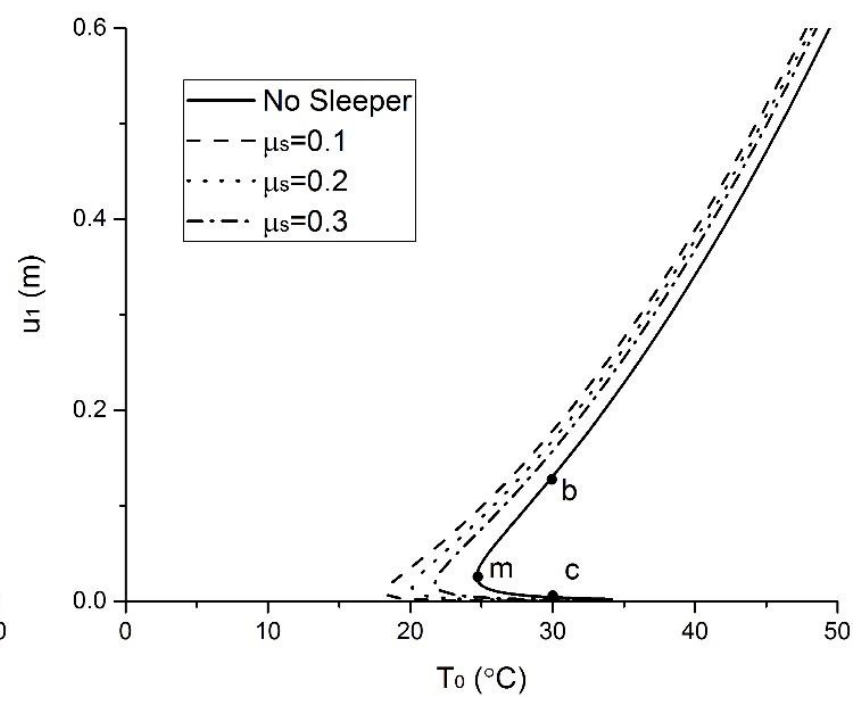

(b)

Fig. 12 Axial expansion displacement $u_{1}$. (a) Different sleeper height $v_{o m} \cdot \mu_{s}=0.1$. (b) Different sleeper friction $\mu_{s} . v_{\text {om }}=0.1 \mathrm{~m}$.

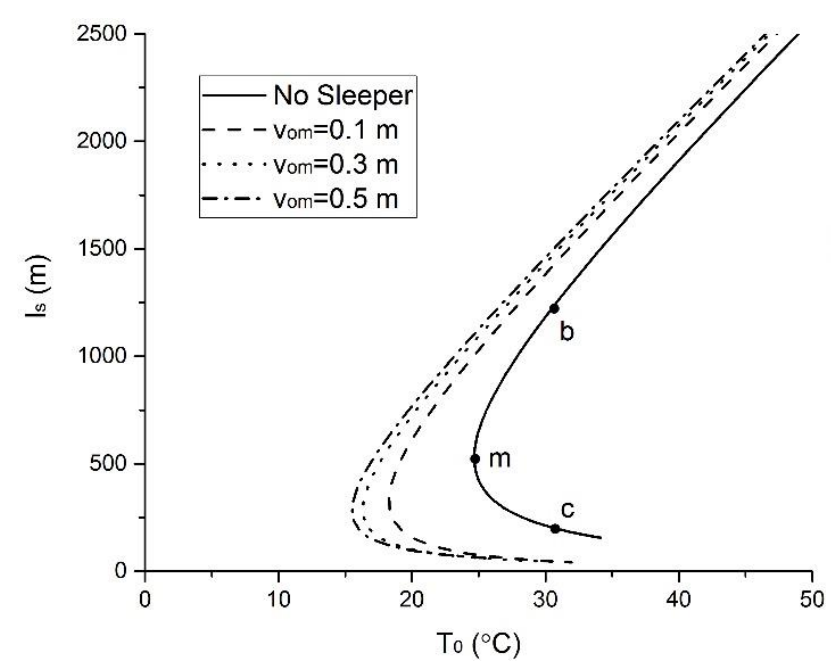

(a)

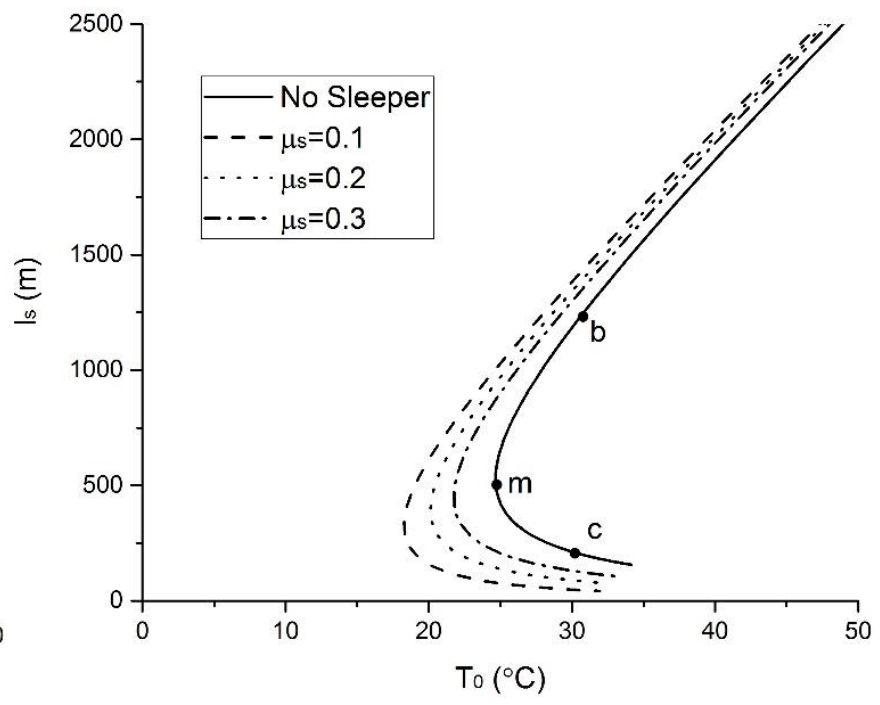

(b)

Fig. 13 Length $l_{s}$ of the feed-in zone. (a) Different sleeper height $v_{o m} . \mu_{s}=0.1$. (b) Different sleeper friction

$$
\mu_{s} . v_{o m}=0.1 \mathrm{~m} \text {. }
$$




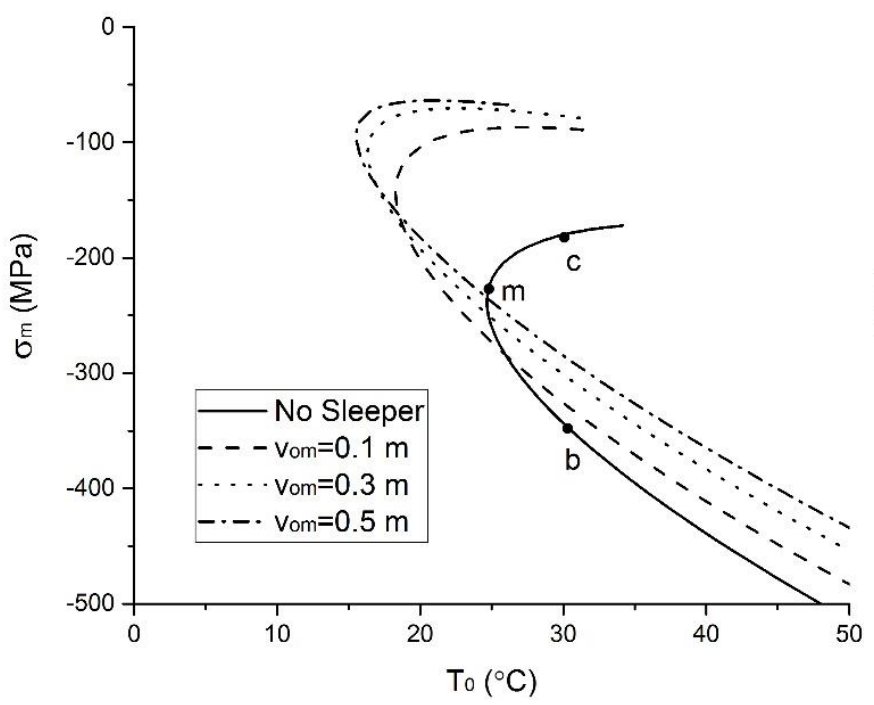

(a)

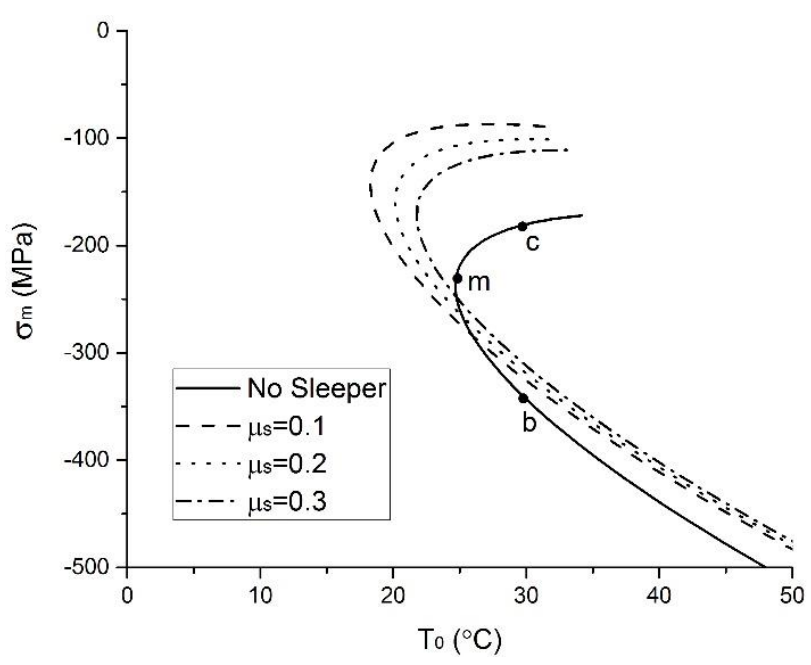

(b)

Fig. 14 Maximum axial compressive stress $\sigma_{m}$. (a) Different sleeper height $v_{o m} \cdot \mu_{s}=0.1$. (b) Different sleeper friction $\mu_{s} . v_{o m}=0.1 \mathrm{~m}$.

The relationships between the maximum axial compressive stress $\sigma_{m}$ and the total temperature difference $T_{0}$ with different values of sleeper height $v_{o m}$ and sleeper friction $\mu_{s}$ are shown in Fig. 14-a and Fig. 14-b, respectively. It is seen from Fig. 14 that the maximum axial compressive stress $\sigma_{m}$ increases (in absolute value) with increasing total temperature difference $T_{0}$ for given sleeper height $v_{o m}$ and sleeper friction $\mu_{s}$. The rate of increase decreases with increasing sleeper height $v_{o m}$. However, the rate of increase for different values of the sleeper friction $\mu_{s}$ stays almost the same. From Fig. 14, the maximum axial compressive stress $\sigma_{m}$ decreases with increasing sleeper height $v_{o m}$ and sleeper friction $\mu_{s}$ under the same temperature difference $T_{0}$, and is smaller than that for a pipeline without sleeper. Thus, increase of sleeper height $v_{o m}$ can be used as a method to reduce the maximum axial compressive stress $\sigma_{m}$ of the pipeline in the post-buckling stage. Consequently, the integrity of the pipeline within the buckle is improved. However, the minimum critical temperature difference will be increased when the sleeper friction $\mu_{s}$ is increased. Moreover, the rate of decrease of the maximum axial compressive stress $\sigma_{m}$ is not affected much by increasing the sleeper friction $\mu_{s}$. Therefore, it is not an appropriate strategy to reduce the maximum axial compressive stress $\sigma_{m}$ by increasing the sleeper friction $\mu_{s}$.

\subsection{Imperfection effect}

The analysis so far has assumed that the profile of the pipeline is perfectly straight in the horizontal plane, namely no horizontal offset. Deviations from a straight profile for pipelines laid on the sleeper are introduced by the pipelaying vessel's sway motion during the installation process. Large lateral offsets may be difficult to implement during installation; only very small offsets are likely during normal installation [35, 39]. The out of-straightness or initial lateral imperfection can lower the safe temperature difference and affect the post-buckling behaviour. Therefore, it is important to investigate the effect of imperfections on the lateral buckling behaviour of pipelines with sleeper. In this analysis, a lateral deflection imperfection is imposed around the location of the sleeper, the configuration of which is the configuration obtained in this paper, namely $w_{1}$ and $w_{2}$. So only the amplitude of the initial imperfection $w_{\text {om }}$ should be applied. There are two possible states for a pipeline initial imperfection: unstressed and stressed pipe. The unstressed pipe corresponds to a local imperfection in the pipe itself, which means the initial state of the pipeline with such an imperfection is unstressed. The stressed pipe represents the case where the unstressed pipeline is straight and where it forms an initial curvature due to the pipe-laying vessel's sway motion or foundation irregularities. So the imperfection included here is the stressed case. For this case, the equations governing the horizontal deflection will not be affected. The effect of the initial imperfection is that an initial geometric shortening $u_{20}$ exists. This 
shortening $u_{20}$ can be calculated by Eq. (26) when the amplitude of initial imperfection $w_{\text {om }}$ is given. So, Eq. (28) should be rewritten as

$$
P_{0}=P+f_{A}\left(\sqrt{\frac{1}{3} l_{1}^{2}+\frac{2 E A\left(u_{2}-u_{20}\right)}{f_{A}}}-\frac{2}{3} l_{1}\right)
$$

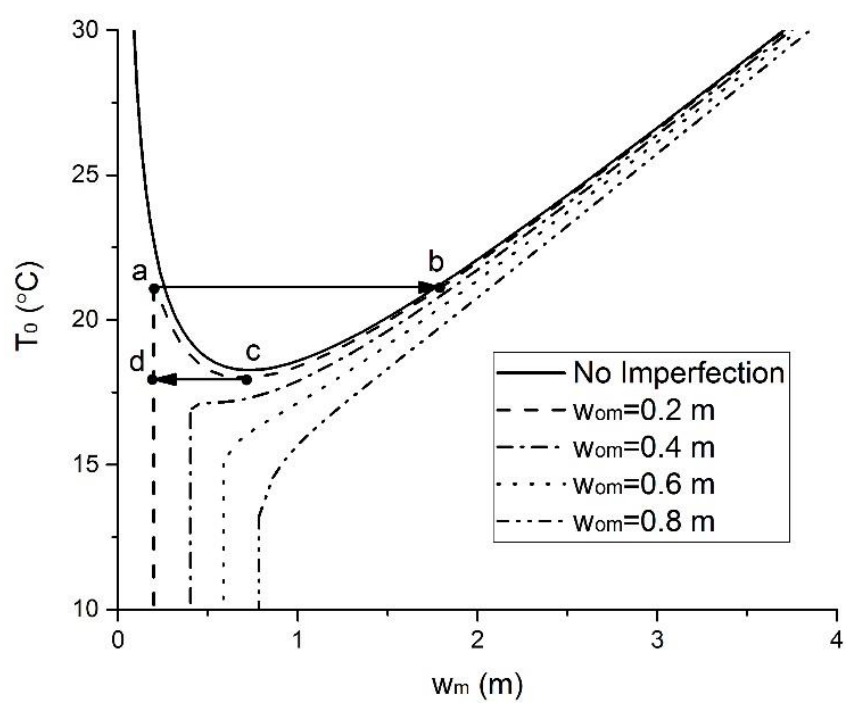

Fig. 15 The effect of imperfections on the load-deflection behavior. Arrows indicate dynamic jumps under increasing (to the right) or decreasing (to the left) $T_{0}$.

The effect of imperfections on the load-deflection behaviour of the pipeline is illustrated in Fig. 15, which shows an enlargement of the region of interest in Fig. 4 with some typical imperfection curves added. From Fig. 15, we see that for smaller values of the imperfection amplitude, such as $w_{\mathrm{om}}=0.2 \mathrm{~m}$, the load-deflection curves have folds where dynamic jumps of the structure may occur under both increasing and decreasing temperature. Taking $w_{\mathrm{om}}=$ $0.2 \mathrm{~m}$ as an example, when the temperature difference increases to $T_{0}(\mathrm{a})$, the pipeline will jump from point $a$ to point $b$ if some disturbance occurs. With further increasing temperature difference, the pipeline will follow the postbuckling path. When the temperature difference decreases from $T_{0}(\mathrm{~b})$ to $T_{0}(\mathrm{c})$, the pipeline will follow the buckling from point $b$ to point $c$. Then, the pipeline will jump from point $c$ back to point $d$. For larger values of the imperfection amplitude, such as $w_{\mathrm{om}}=0.8 \mathrm{~m}$, the snap-through phenomenon disappears.

\subsection{Error analysis and validation}

The main approximation we make in our model (apart from the assumption of small deflections) is that we calculate the lateral buckling solution for constant compressive force $P$ (cf. Eq. (12)), an approximation generally made in the literature (e.g., [10]). In doing this we ignore the extra contributions to the pressure for $l_{1}<x<l_{2}$ in Eq. (9). However, it is good to stress that we make this approximation only in calculating the shape of the lateral buckling solution and not in the computation of the corresponding temperature difference (based on deformational compatibility) and not in the energy analysis. We shall call this Case a.

In order to investigate the error incurred by this constant $P$ approximation, we also calculate the lateral buckling solution with constant compressive force $P+f_{A t}+f_{A}\left(l_{2}-l_{1}\right)$ (Case b). Since this is the maximum compressive force attained in the buckled region, Case $\mathrm{b}$ will give us an upper bound to the error. The lateral displacement amplitude and maximum axial compressive stress of Case $\mathrm{b}$ are denoted by $w_{m b}$ and $\sigma_{m b}$, respectively. The two cases are compared in Fig. 16 and Fig. 17. Fig. 16 shows that the difference between Case a and Case b is very small, the critical temperatures being $15.515{ }^{\circ} \mathrm{C}$ and $15.185{ }^{\circ} \mathrm{C}$, respectively (an error of $2.13 \%$ ). From Fig. 17 we see that the error of lateral displacement amplitude and maximum axial compressive stress between Case a and Case $\mathrm{b}$ is large around the critical temperature, but decreases rapidly to $-3 \% \sim 3 \%$ away from the critical temperature. The large 
relative error near criticality is natural since the lateral deflection is so small. It causes no problems in practice as the absolute stress levels involved are very low (see Fig. 16).

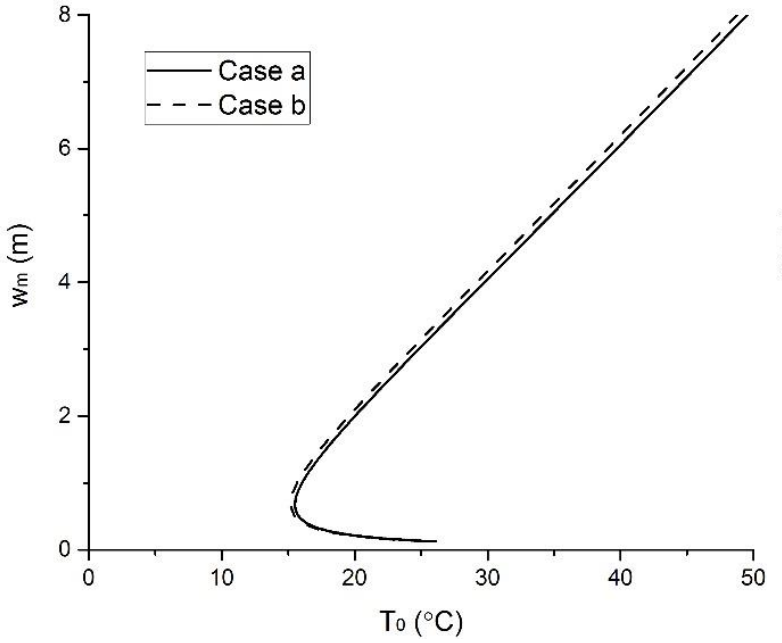

(a)

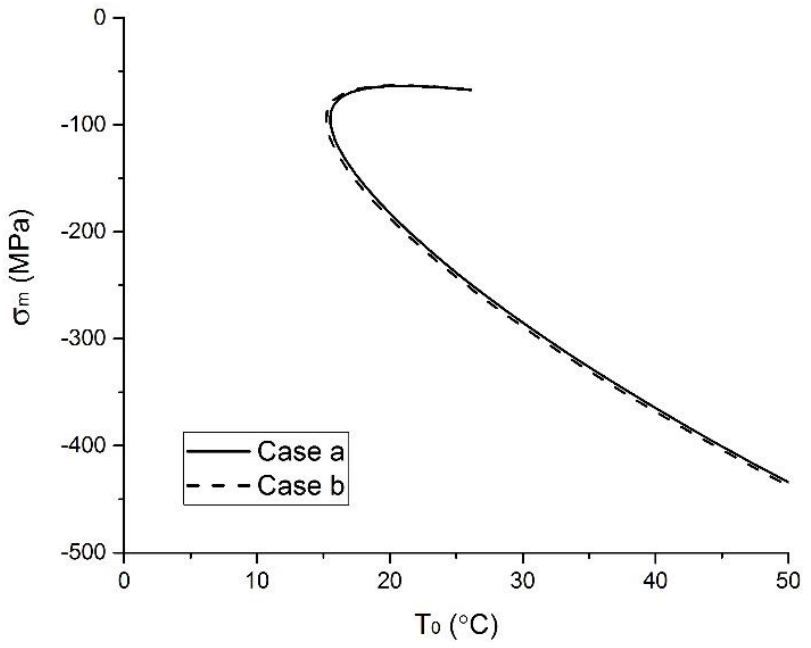

(b)

Fig. 16 Comparison of Case a and Case b. (a) Lateral displacement amplitude. (b) Maximum axial compressive stress.

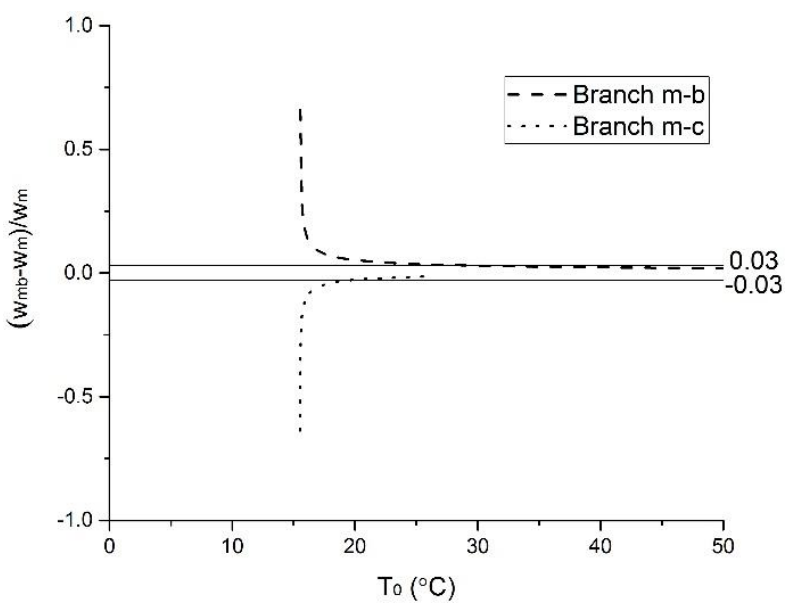

(a)

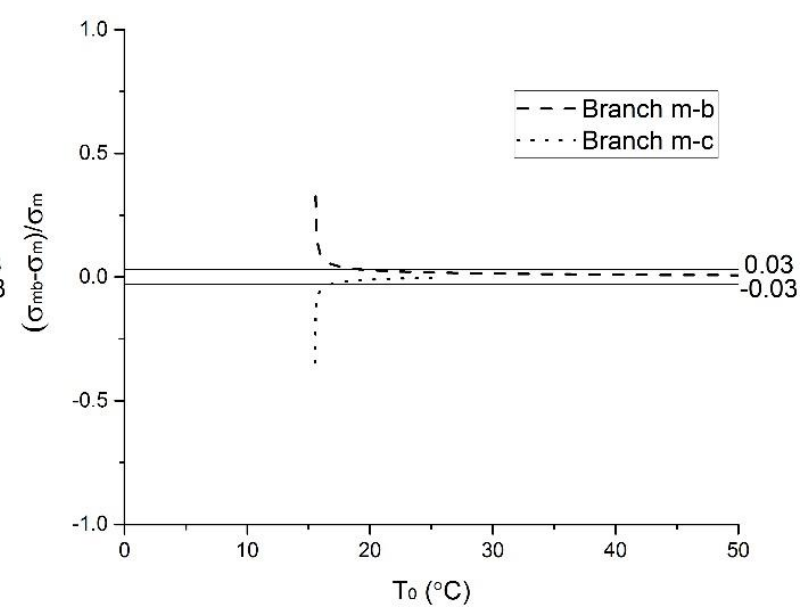

(b)

Fig. 17 The error between Case a and Case b. (a) $\left(w_{m b}-w_{m}\right) / w_{m}$. (b) $\left(\sigma_{m b}-\sigma_{m}\right) / \sigma_{m}$.

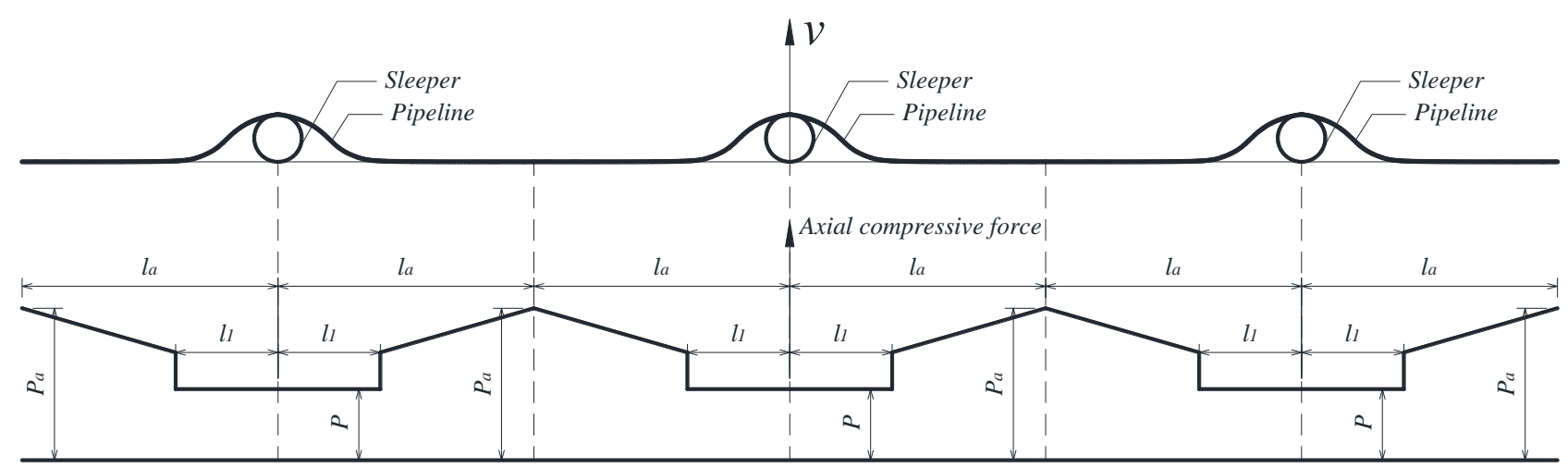

Fig. 18 Pipeline with three sleepers and the corresponding axial compressive force distribution. 


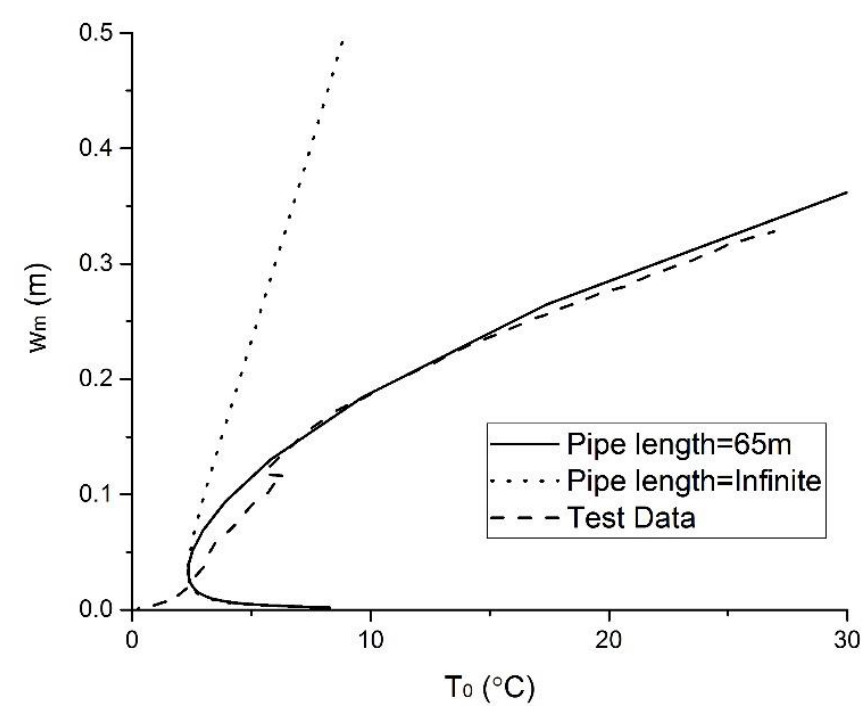

Fig. 19 Validation with test data.

In order to validate the analytical results proposed in this paper, another case is calculated by using the test parameters listed in Table 2. These parameters are the same as those used in the reduced-scale model in [25]. The total pipe length in [25] was $195 \mathrm{~m}$. Three sleepers with equal spacing of $2 l_{a}=65 \mathrm{~m}$ were installed to trigger lateral buckling, as shown in Fig. 18. $l_{a}$ represents the maximum axial feed-in length. $P_{a}$ is the axial compressive force at the virtual anchors between two buckles. By symmetry, the axial feed-in displacement at the midpoint between two sleepers, namely at the virtual anchor between two buckles, is zero. So, for comparison with our model, we only consider a pipeline section over one sleeper and of length $L=65 \mathrm{~m}$. For this case, the maximum axial feed-in length $l_{a}$ is $32.5 \mathrm{~m}$. When the feed-in length $l_{s}$ is smaller than $l_{a}$, so that two adjacent buckles triggered by two adjacent sleepers are independent, all the formulae derived in this paper can be applied. When $l_{s}$ is larger than $l_{a}$, the formula for axial compressive force should be modified due to the limit of axial feed-in length. In that case, by axial force balance, we have

$$
P_{a}=P+f_{A}\left(l_{a}-l_{1}\right)+f_{A t}
$$

and the length of axial expansion within the pipeline section $0<x<l_{a}$ should be modified to

$$
u_{1}=\int_{0}^{l_{l}} \frac{\Delta \bar{P}(x)}{E A} d x
$$

where

$$
\Delta \bar{P}(x)= \begin{cases}P_{0}-P & 0<x<l_{1} \\ f_{A}\left(l_{a}-x\right) & l_{1}<x<l_{a}\end{cases}
$$

Then, given $T_{0}$ and $l_{a}$, Eq. (26), Eq. (29), Eq. (41), Eq. (42) and (with Eq. (14) and Eq. (15) inserted) can be solved in conjunction with Eq. (16) and Eq. (17) to obtain $P, l_{2}$ and the coefficients $A_{1}-A_{4}$ and $B_{1}-B_{4}$, and hence the lateral deflection $w_{1}, w_{2}$.

The results from model test and analytical method are compared in Fig. 19. The present analytical results appear to agree very well with the test data. For comparison, we also include in Fig. 19 results (labelled 'Pipe length=infinite') obtained by assuming that always $l_{s}<l_{a}$, i.e., that the required feed-in length $l_{s}$ is always available. We see that the lateral displacement amplitude $w_{m}$ for a pipe of length $65 \mathrm{~m}$ is much smaller than that for a pipe of 'infinite length'. This explains why many sleepers should be installed at regular intervals to trigger pipeline buckling at several planned locations. 
Table 2. Test parameters

\begin{tabular}{ccc}
\hline Parameters & Values & Unit \\
\hline External diameter $D$ & 15 & $\mathrm{~mm}$ \\
Wall thickness $t$ & 0.9 & $\mathrm{~mm}$ \\
Elastic modulus $E$ & 191 & $\mathrm{GPa}$ \\
Pipeline submerged weight & 3.94 & $\mathrm{~N} / \mathrm{m}$ \\
Coefficient of thermal expansion $\alpha$ & $1.75 \times 10^{-5}$ & $/{ }^{\circ} \mathrm{C}$ \\
Lateral friction coefficient $\mu_{L}$ & 0.7 & --- \\
Axial friction coefficient $\mu_{A}$ & 0.7 & --- \\
Sleeper friction $\mu_{S}$ & 0.1 & --- \\
Sleeper height $v_{\text {om }}$ & 0.03 & $\mathrm{~m}$ \\
Pipe length $L$ & 65 & $\mathrm{~m}$ \\
\hline
\end{tabular}

\section{Conclusions}

We have derived analytical solutions for the lateral buckling of unburied subsea pipelines with sleeper. The solutions are based on small-deflection (beam) theory, but take exact account of the compatibility between axial and lateral deformation to obtain curves of lateral deflection and axial compressive stress against temperature difference $T_{0}$.

From our parameter studies the following conclusions can be drawn:

(i) The energy analysis reveals several critical temperatures for pipelines with and without sleeper (see Fig. 4). No lateral buckling solutions exist for temperatures less than $T_{m}$, which therefore represents an upper bound to safe operating temperatures for the pipeline. For temperatures larger than $T_{m}$ two solutions with sleeper are available. Initially these have larger energy than the unbuckled pipe. However, we find that, typically, for only slightly higher temperatures $\left(T_{0}>T_{e}\right)$ one of the solutions acquires an energy lower than that of the trivial solution (see Fig. 5). For such temperatures, the unbuckled pipe with sleeper can therefore be considered unstable under sufficiently large perturbations (e.g., dynamic disturbances due to irregular fluid flow through the pipe or earthquakes). Meanwhile, the pipeline without sleeper becomes similarly unstable under large perturbations at temperatures slightly larger than the higher temperature $T_{n}$. This instability, however, is sudden and may occur anywhere along the pipe. Thus, by introducing a controlled deformation at a specific location, a sleeper causes the pipeline to buckle laterally at lower temperature $T_{0}$, thereby avoiding a potentially hazardous instability.

(ii) For pipelines with sleeper, under the same operating temperature difference, both the buckled region and the lateral deflection of the buckled pipeline with sleeper are larger than those without sleeper, and both grow with increasing sleeper height and decreasing sleeper friction. Also, the maximum bending stress at the midpoint of the pipeline with sleeper is smaller than that without sleeper, and further decreases with increasing sleeper height. However, the sleeper friction does not have much effect on the bending stress.

(iii) The minimum critical temperature difference decreases with increasing sleeper height for given sleeper friction and increases with increasing sleeper friction for given sleeper height, and is smaller than that of pipelines without sleeper. Thus, it is an appropriate strategy to increase the sleeper height or to decrease the sleeper friction to reduce the minimum critical temperature difference.

(iv) Under the same temperature difference, the lateral displacement amplitude increases and the maximum axial compressive stress decreases with increasing sleeper height. However, both the lateral displacement amplitude and the maximum axial compressive stress decrease with increasing sleeper friction. The maximum axial compressive stress increases with increasing total temperature difference for given sleeper height and sleeper friction and the rate of increase decreases with increasing sleeper height. However, the rate of increase for different values of the sleeper 
friction stays almost the same. Thus, it is an appropriate strategy to reduce the maximum axial compressive stress by increasing the sleeper height.

(v) For relatively small values of the imperfection amplitude, a snap-through instability occurs, which disappears for sufficiently large imperfection amplitude.

(vi) We consider the case of a single sleeper, but in Section 3.5 indicate how the model can be extended to multiple sleepers. This involves putting a maximum on the available feed-in length $l_{s}$ given by the spacing of the sleepers. The use of multiple sleepers reduces the maximum deflection and the maximum compressive stress of the pipeline, so when applied to pipelines with multiple sleepers our model is less affected by the limitations of the small-deflection assumption.

\section{Acknowledgments}

The authors would like to acknowledge that the work described in this paper was funded by the National Key Basic Research Program of China (2014CB046805).

\section{Appendix A.}

$$
\begin{aligned}
& A_{1}=\frac{\sec \left(\lambda l_{2}\right)\left(f \cos \left(\lambda\left(l_{1}-l_{2}\right)\right)-f-f_{o w} \lambda \sin \left(\lambda l_{2}\right)+f_{t} \lambda \sin \left(\lambda\left(l_{1}-l_{2}\right)\right)\right)}{E I \lambda^{4}} \\
& A_{2}=\frac{f_{o w}}{E I \lambda^{3}} \\
& A_{3}=-\frac{f_{o w}}{E I \lambda^{2}} \\
& A_{4}=\frac{f\left(l_{1}-l_{2}\right)^{2}+2 f_{o w} l_{2}+2 f_{t}\left(l_{2}-l_{1}\right)}{2 E I \lambda^{2}} \\
& B_{1}=-\frac{\tan \left(\lambda l_{2}\right)\left(-f \sin \left(\lambda l_{1}\right)+f_{o w} \lambda+f_{t} \lambda \cos \left(\lambda l_{1}\right)\right)+f \sec \left(\lambda l_{2}\right)}{E I \lambda^{4}} \\
& B_{2}=\frac{-f \sin \left(\lambda l_{1}\right)+f_{o w} \lambda+f_{t} \lambda \cos \left(\lambda l_{1}\right)}{E I \lambda^{4}} \\
& B_{3}=-\frac{-f l_{1}+f_{o w}+f_{t}}{E I \lambda^{2}} \\
& B_{4}=\frac{\lambda^{2} l_{2}\left(2\left(-f l_{1}+f_{o w}+f_{t}\right)+f l_{2}\right)+2 f}{2 E I \lambda^{4}}
\end{aligned}
$$

\section{References}

[1] D. Bruton, D.J. White, C.Y. Cheuk, M.D. Bolton, M. Carr, Pipe-soil interaction behavior during lateral buckling, including large-amplitude cyclic displacement tests by the SAFEBUCK JIP, in: Offshore Technology Conference 2006, pp. 1-20.

[2] H.R.C. Dingle, D.J. White, C. Gaudin, Mechanisms of pipe embedment and lateral breakout on soft clay, Can. Geotech. J., 45 (2008) 636-652.

[3] R. Shi, L. Wang, Single Buoyancy Load to Trigger Lateral Buckles in Pipelines on a Soft Seabed, Journal of Engineering Mechanics, (2015).

[4] R. Reddy, Lateral Buckling Behaviour of Snake-Lay Pipeline With Vertical Support at Crown, in: International Conference on Ocean, Offshore and Arctic Engineering, 2013.

[5] F. Sinclair, M. Carr, D. Bruton, T. Farrant, Design Challenges and Experience With Controlled Lateral Buckle Initiation Methods, in: International Conference on Ocean, Offshore and Arctic Engineering, Honolulu, Hawaii, USA, 2009.

[6] Y. Urthaler, R. Watson, J. Davis, Lateral Buckling of Deepwater Pipelines in Operation, in: International Conference on Ocean, Offshore and Arctic Engineering, Rio de Janeiro, Brazil, 2012. 
[7] R. Peek, H. Yun, Flotation to trigger lateral buckles in pipelines on a flat seabed, Journal of Engineering Mechanics, 4 (2007) 442-451.

[8] G. Harrison, M. Harrison, D. Bruton, King flowlines-thermal expansion design and implementation, in: Offshore Technology Conference, Offshore Technology Conference, 2003.

[9] R. Peek, N.Ø. Kristiansen, Zero-radius bend method to trigger lateral buckles, Journal of Transportation Engineering, 135 (2009) 946-952.

[10] R.E. Hobbs, In-service buckling of heated pipelines, Journal of Transportation Engineering, 110 (1984) $175-189$.

[11] N. Taylor, A.B. Gan, Submarine pipeline buckling-imperfection studies, Thin-Walled Structures, 4 (1986) 295-323.

[12] G.T. Ju, S. Kyriakides, Thermal buckling of offshore pipelines, Journal of Offshore Mechanics and Arctic Engineering, 110 (1988) 355-364.

[13] N. Taylor, V. Tran, Prop-imperfection subsea pipeline buckling, Marine Structures, 6 (1993) 325-358.

[14] H. Karampour, F. Albermani, J. Gross, On lateral and upheaval buckling of subsea pipelines, Engineering Structures, 52 (2013) 317-330.

[15] R. Liu, W. Liu, X. Wu, S. Yan, Global lateral buckling analysis of idealized subsea pipelines, Journal of Central South University, 21 (2014) 416-427.

[16] Z. Hong, R. Liu, W. Liu, S. Yan, Study on lateral buckling characteristics of a submarine pipeline with a single arch symmetric initial imperfection, Ocean Eng., 108 (2015) 21-32.

[17] J.G.A. Croll, A simplified model of upheaval thermal buckling of subsea pipelines, Thin-Walled Structures, 29 (1997) 59-78.

[18] L. Wang, R. Shi, F. Yuan, Z. Guo, L. Yu, Global buckling of pipelines in the vertical plane with a soft seabed, Applied Ocean Research, 33 (2011) 130-136.

[19] R. Shi, L. Wang, Z. Guo, F. Yuan, Upheaval buckling of a pipeline with prop imperfection on a plastic soft seabed, Thin-Walled Structures, 65 (2013) 1-6.

[20] X. Zeng, M. Duan, Mode localization in lateral buckling of partially embedded submarine pipelines, International Journal of Solids and Structures, 51 (2014) 1991-1999.

[21] J. Zhu, M.M. Attard, D.C. Kellermann, In-plane nonlinear localised lateral buckling of straight pipelines, Engineering Structures, 103 (2015) 37-52.

[22] Z. Wang, G.H.M. van der Heijden, Localised lateral buckling of partially embedded subsea pipelines with nonlinear soil resistance, Thin-Walled Structures, (2017).

[23] T.C. Maltby, C.R. Calladine, An investigation into upheaval buckling of buried pipelines-I. Experimental apparatus and some observations, International Journal of Mechanical Sciences, 37 (1995) 943-963.

[24] T.C. Maltby, C.R. Calladine, An investigation into upheaval buckling of buried pipelines-II. Theory and analysis of experimental observations, International Journal of Mechanical Sciences, 37 (1995) 965-983.

[25] C. de Oliveira Cardoso, R.F. Solano, Performed of Triggers to Control Thermal Buckling of Subsea Pipelines Using Reduced Scale Model, in: International Offshore and Polar Engineering Conference, International Society of Offshore and Polar Engineers, 2015.

[26] H.C. Silva-Junior, C.O. Cardoso, M.A.P. Carmignotto, J.C. Zanutto, Reduced Model Device of Solutions to Control Thermal Buckling Effects in HP-HT Subsea Pipelines, in: International Conference on Ocean, Offshore and Arctic Engineering, 2009.

[27] H. Karampour, F. Albermani, Experimental and numerical investigations of buckle interaction in subsea pipelines, Engineering Structures, 66 (2014) 81-88.

[28] H. Karampour, F. Albermani, M. Veidt, Buckle interaction in deep subsea pipelines, Thin-Walled Structures, (2013).

[29] Z. Wang, Z. Chen, H. Liu, Y. Bu, Static and dynamic analysis on upheaval buckling of unburied subsea pipelines, Ocean Eng., 104 (2015) 249-256. 
[30] Z. Wang, Z. Chen, H. Liu, Numerical study on upheaval buckling of pipe-in-pipe systems with full contact imperfections, Engineering Structures, 99 (2015) 264-271.

[31] R. Liu, H. Xiong, X. Wu, S. Yan, Numerical studies on global buckling of subsea pipelines, Ocean Eng., 78 (2014) $62-72$.

[32] R. Liu, P. Basu, H. Xiong, Laboratory tests and thermal buckling analysis for pipes buried in Bohai soft clay, Marine Structures, 43 (2015) 44-60.

[33] Z. Hong, R. Liu, W. Liu, S. Yan, A lateral global buckling failure envelope for a high temperature and high pressure (HT/HP) submarine pipeline, Applied Ocean Research, 51 (2015) 117-128.

[34] X. Zhang, M. Duan, Prediction of the upheaval buckling critical force for imperfect submarine pipelines, Ocean Eng., 109 (2015) 330-343.

[35] X. Zeng, M. Duan, X. Che, Critical upheaval buckling forces of imperfect pipelines, Applied Ocean Research, 45 (2014) 33-39.

[36] Z. Wang, Y. Tanga, C. Wang, Analytical solution for lateral buckling of unburied subsea pipelines with distributed buoyancy section, Ocean Eng., (2017).

[37] B.R. Antunes, R.F. Solano, M.A. Vaz, Analytical formulation of distributed buoyancy sections to control lateral buckling of subsea pipelines, in: International Conference on Ocean, Offshore and Arctic Engineering, 2010.

[38] G. Li, L. Zhan, H. Li, An analytical solution to lateral buckling control of subsea pipelines by distributed buoyancy sections, Thin-Walled Structures, 107 (2016) 221-230.

[39] Q. Bai, X. Qi, M. Brunner, Global Buckle Control with Dual Sleepers in HP/HT Pipelines, 2009.

[40] DNV-RP-F105, FREE SPANNING PIPELINES, in, 2006. 